\title{
Software Project Contracts and Scheduling by GRGA and EVM
}

\author{
Dinesh Bhagwan Hanchate \\ Comp. Engg. Deptt. \\ V.P.'s College Of Engg., \\ Baramati, Pune
}

\author{
Rajankumar S. Bichkar \\ Prof. ( E \& Tc ) and Dean \\ G. H. R. C. O. E. M., Wagholi, \\ Pune, India.
}

\begin{abstract}
The well planned SPLC (Software project life cycle) doesn't give certainty about the completion of project in time and budget. The PMP (Project Management Processes), pregnant processes, rework, some float types and review process, always, put stumbling block for completion of project in Time. Apart from this defined process, there is need of genius decision making process. The various planned schedule, redundancy and contingency target schedules can be used as input for decision making processes and the solutions to be adapted during the stages of SPLC. This paper gives various schedule types with respect to software project contracts. These schedule types are the outputs of GRGA (Gene Repair Genetic algorithm) along the utilisation of EVM (Earned Value Management) concepts. The GRGA (Gene Repair based Genetic Algorithm) approach gives choice to change the constraints and or features as objective components in objective function. In this paper, we present different schedules as the outcome to evaluate the effectiveness of genetic operator GeneRepair. This operator is developed to correct invalid schedule generated following crossover and mutation. Following implementation and testing of GA with GeneRepair, we found a significant positive side in our results in speed and accuracy also. we have been able to generate very good results in an efficient manner, in terms of both time and number of evaluations using GeneRepair with traditional crossover and mutation operators.
\end{abstract}

\section{General Terms:}

Software Project Management, Machine learning

\section{Keywords:}

PMP, Software Contacts, GRGA, COCOMO, SPSP (Software Project Scheduling problem), Constraints Optimization, EVM.

\section{INTRODUCTION}

Cognizant engineers and software managers are often in the business of scheduling a series of engineering task. In constructing good schedules, management requires satisfactory schedule plan. The standards algorithms are too consuming for any practical use for getting an optimal schedule. A machine learning technique called genetic algorithm is iterative, Meta-heuristic search method which gives the near optimal solution to a problem. GRGA [24] approach is considered, in this study, to get and generate various schedules for software engineering project for various combination of features considered under the different circumstances of ACWP (Actual Cost of Work Performed [1]). These schedule plans or types are related with time constraint, cost constraint, with timecost-skill constraint, time- cost skill-overloading constraint and others plans. This paper imparts various near optimal schedules which give alternative schedule choice to PM to adapt any solution according to the situation and constraints required by project scope and concerned contract.

This paper addresses some issues, these are

* Study of Software contracts, PMP, scheduling plan \& estimation [2] [47].

* EVA used in proposed approach based on EVM by Anita [45], Hughes [11] and SEE principles by Bohem principle

$\star$ Importance of Gene repair.

$\star$ pros and cons of GRGA and COCOMO-II solutions.

* The work is studied and derived from that of [ Carl 2001] [9], we do take opportunity to do the experiments for comparison and similarities with proposed approach by adding some features.

The paper is arranged as follows

- section I gives introduction part.

- section II gives the theory and Hypothesis for proposed GRGA approach and PMP [1].

- section III discusses about GA, Gene Repair and proposed GRGA.

- section IV puts light on assumptions and problem definition for Proposed approach.

- section V gives study of various schedule types.

- section VI describes results and discussion.

- section VII gives future direction along with conclusion.

- section VIII is about concerned reading, work, inspiration and support by Machine learning related authors.

\section{THE THEORY AND HYPOTHESIS}

\subsection{Project Management Processes [4]}

Main development part of SDLC starts with the initializing the processes and it's group. Monitoring and Controlling processes are measuring the significance performance project deviation in terms of schedule. The costs of the schedule perform the related 
Table 1. Notations, symbols and meanings [45] [18] [17].

\begin{tabular}{|c|c|}
\hline Notations & Meaning \\
\hline WBS & Work Breakdown Structure \\
\hline MM & man-months \\
\hline FSP & Full Time software professional \\
\hline KDSI & $\begin{array}{l}\text { Thousands of delivered } \\
\text { source instructions }\end{array}$ \\
\hline TDEV & Development time \\
\hline $\mathrm{HC}_{T_{i}}$ & Head Count \\
\hline SPLC & Software Project Life Cycle \\
\hline $\mathrm{CD}_{T_{i}}$ & $\begin{array}{l}\text { COCOMO Calculated duration } \\
\text { of task } \mathrm{T}_{i}\end{array}$ \\
\hline $\mathrm{GROD}_{T_{i}}$ & $\begin{array}{l}\text { Optimistic duration of task } \mathrm{T}_{i} \\
\text { obtained by GRGA approach }\end{array}$ \\
\hline $\mathrm{CPD}_{T_{i}}$ & $\begin{array}{l}\text { COCOMO pessimistic duration } \\
\text { of task } \mathrm{T}_{i} \text { and equal to } 1.6 \times \mathrm{CD}\end{array}$ \\
\hline DUR & Project duration \\
\hline $\mathrm{EDUR}_{\text {avg }}$ & $\begin{array}{l}\text { Expected project DUR } \\
\doteq \mathrm{CED}_{T_{i}}\end{array}$ \\
\hline TPHC & $\frac{1}{\text { Total project head count. }}$ \\
\hline EMF & Effort multiplier factors \\
\hline $\mathrm{s}^{t h}$ & Schedule number in generation \\
\hline $\mathrm{P}_{c}$ & Crossover rate \\
\hline $\mathrm{P}_{m}$ & Mutation rate \\
\hline $\mathrm{FC}_{s}$ & Fitness of $\mathrm{s}^{t h}$ Schedule \\
\hline $\mathrm{P}_{s}$ & Total Penalty of $\mathrm{s}^{t h}$ schedule \\
\hline SP & Size of Population \\
\hline $\mathrm{SalE}_{E_{j}}$ & $\begin{array}{l}\text { Maximum salary of employee } \\
\text { among all employees }\end{array}$ \\
\hline $\mathrm{TT}_{s}$ & Total time of $\mathrm{s}^{t h}$ schedule \\
\hline $\mathrm{OT}_{s}$ & $\begin{array}{l}\text { Over time of } s^{t h} \text { schedule } \\
\text { considered as penalty }\end{array}$ \\
\hline $\mathrm{TC}_{s}$ & $\begin{array}{l}\text { Number of Task completion of } \\
\mathrm{s}^{t h} \text { schedule }\end{array}$ \\
\hline GRDLC $_{s}$ & DLC of $s^{t h}$ schedule by GRGA \\
\hline $\mathrm{CDLC}_{s}$ & DLC of $\mathrm{s}^{t h}$ schedule by COCOMO \\
\hline$\mu$ & Overtime penalty rate \\
\hline$\eta$ & Time penalty rate \\
\hline $\mathrm{TP}_{s}$ & $\begin{array}{l}\text { Time Penalty } \\
\text { of } \mathrm{s}^{t h} \text { schedule }\end{array}$ \\
\hline $\mathrm{TCHC}_{s}$ & $\begin{array}{l}\text { Total COCOMO head count } \\
\text { of } \mathrm{s}^{t h} \text { schedule }\end{array}$ \\
\hline $\mathrm{GRSHD}_{s}$ & $\begin{array}{l}\text { Schedule obtained by GRGA } \\
\text { approach of } \mathrm{s}^{t h} \text { schedule }\end{array}$ \\
\hline CPSHD $_{s}$ & COCOMO pessimistic of $\mathrm{s}^{t h}$ schedule \\
\hline $\mathrm{COSHD}_{s}$ & COCOMO optimistic of $\mathrm{s}^{t h}$ schedule \\
\hline SPI & Schedule Performance Index \\
\hline SV & Schedule Variance \\
\hline $\mathrm{CB}$ & Cost Baseline \\
\hline CPI & Cost performance Index \\
\hline BAC & Budget at completion \\
\hline CEVP & Cost Estimate Validation Process \\
\hline
\end{tabular}

ICC (Integrated Change Control) activities. Ultimately at the end,the SM (Software Manager) /PM will check the total and entire completed work. He confirms the objectives are met or not; this can be achieved by the various studies of schedule types which is produced here by EVM (Earned Value Management [11] [6]). According to PMBOK, there are five Project Management Processes [1], are must for any type of project. These five Project
Table 2. Notations,symbols and meanings [45] [18] [17].

\begin{tabular}{|l|l|}
\hline Notations & Meaning \\
\hline CMP & Cost Management Plan \\
\hline AC & Actual Cost \\
\hline EV & Earned value or approved or budgeted cost \\
\hline CPF & Cost-plus-fee \\
\hline CPFP & Cost-plus-fixed fee \\
\hline CPIF & Cost-plus-incentive-fee \\
\hline PEV & Proposed Earned value \\
\hline CEV & COCOMO Earned Value \\
\hline GREV & EV obtained by GRGA Approach \\
\hline AC & Actual Cost \\
\hline PDM & Precedence Diagramming Method \\
\hline SCE & Software Cost Estimation \\
\hline PEV & Proposed EV \\
\hline CEV & COCOMO-EV \\
\hline CTT & COCOMO Threshold Time \\
\hline ET & Estimated Time \\
\hline SM & Schedule Margine \\
\hline CM & Cost Margine \\
\hline SHD & $\begin{array}{l}\text { the post fix word SHD in some notations } \\
\text { give array of tasks assignment to employees. }\end{array}$ \\
\hline GRD & Total time of s ${ }^{\text {th }}$ GRGA schedule \\
\hline Total Time & $\begin{array}{l}\text { Total time (in figs. and tables) } \\
\text { is GRD except simple time ST-2 type }\end{array}$ \\
\hline GRCT & $\begin{array}{l}\text { Critical time by GRGA } \\
\text { GRCT is CT obtained and shown in } \\
\text { respective schedule sections in figures }\end{array}$ \\
\hline Pr & profit \\
\hline C & Constant \\
\hline TO & Turnover \\
\hline Prior & History of clients \\
\hline CDC & Cost oriented contract \\
\hline TDC & Time oriented Contract. \\
\hline & \\
\hline & \\
\hline
\end{tabular}

Management Processes are though different in nature with any Project Phases life Cycles as these processes are dependent on industry.

These processes can be embedded in every stages of SDLC [30]. These five groups become important part of the SDLC. The MPG (Monitoring Process Group) is responsible for potential performance of projects in terms of schedule and costs. Hence, the proposed approach uses this group for suitable schedule type.

Any project starts with the Initiating Process Group where it is decided if the project will be selected and accepted based on highlevel planning efforts performed at this stage. If the project is approved, it will move further to the Planning Process Group, so detailed Project Management Plans are prepared. The Executing Process Group will follow, so the work will be completed according to the plans. The results will be supplied to the Monitoring and Controlling Process Group that will make sure the project is on the track in terms of scope, time, cost, risk and quality. In the case when variations to the plan are encountered, depending on the severity of the identified issues, the project will return back to Initiating, Planning or Executing. Following part of the section explains briefly the all processing groups.

2.1.1 IPG (Initiating process group). Any phase of SLDC starts with the IPG. IPG is an iterative clarification of the high level 
users requirements and their needs, objectives, high level costumor needs, summary BAC, milestones, risks and so on, so forth. All these items are the parts of the project charter. The change in above items in turn affects on several components. But, these components can be managed as distinct and uniform projects since it has separate requirements, budgets and cost. Even though the collecting the requirements usually is part of planning phase, the requirements elaboration, specification and clarification stage start actually, here, in order to make available for the charter. The nonfunctional and functional requirements, packages, tools and delivery dates are decided in this group.

2.1.2 PPG (Planning Process Group). Once the approval is given to the charter document, the development will propagate from IPG to the Planning step. From this point, the analysis starts by identifying the domain and scope of the individual projects. This stage finalise the actions to be performed in order to satisfy and meet the requirements, so the approved PMP (project management plans) will be in hand. These PMPs include requirements refinement, BAC, quality requirements, risk identifications, defined scope, WBS creation, schedules, response planning etc.

The analysis part generates a unique and Integrated SRS (Software Requirement Specification) document (at the program level) which describes the use cases with actors and processes. This document is very useful as basis for generation of the test cases. Right after this, the WBS creation will start to make them detailed at feature level. The analysis part will be then continued with a detail and comple te clarification of requirements. A SRS dedicated document will be created for each delivery for designing the test cases. This documents split the features by generating HLD (High Level Design) documents. so, at the end, the WBS latest will be filled in.

2.1.3 EPG (Executing Process Group). The execution activities is performed at the Project and Delivery level. The project will begin with the first Delivery, and followed by second Delivery. This continues with number of deliveries in the projects. Once a component is entirely implemented and seen, it will be prorogated further for the the inspection and validation. Even though an intermediate tests are conducted for QC, but these tests cannot be replacement of the full inspection of delivery package.

2.1.4 CPG (Controlling Process Group). 1.Project Level : Quality Control will be performed at this level, so a strong and stringent quality check will take place for each and every delivery (each completion of milestones) of a project. So, these are managed at this level. The Quality Control Department has role of assembling the individual components into a single package corresponding to the entire project delivery. This is called as integration of project. Integration testing activities will generate Test Results, Test Evaluation Reports and other documents, too, if needed.

2.Program Level: A second QC (Quality Control) step will be performed at this level, in order to confirm the validation of packages of each project are really working bilaterally and together as a whole. Some packages which dosn't pass QC test may be rejected, so they will send back to Executing or Planning stages depending upon the results of test.

2.1.5 MPG (Monitoring Process Group) [32] [45]. In order to see the potential performance deviation of its corresponding projects in terms of schedule and costs, by starting from the following values the EVM (Earned Value Management) method is applied: EV (Earned Value), PV (Planned Value), AC (Actual Cost). The Schedule Variance (SV), Schedule Performance Index (SPI), Cost Variance (CV) and Cost Performance Index (CPI) are useful for measuring the deviations of project deviations in terms of cost and schedule, so these indicators are useful and can be used to show how the project takes path with respect to contract with the client.

2.1.6 CLPG (Closing Process Group). Project closing assists to PMs to make assurance of all the program work is completed. It also make sure that objectives for the projects have met right from starting of the charter and project management plans. All the changes and updation must be reflected in the entire program technical and non technical documentation, including the plans. The updated and historical information, record and statistics should be documented as these files for program and projects archived can be useful for future use. The acceptance of customer should be included, too, as well as the formal completion of project documentation. Finally and last, the project or and process improvement ideas is collected from the stakeholders. The program teams will be informed and communicated about the end of the project, also, the releasing of future new assignments.

\subsection{Types of Contracts [3] [51]}

There is a need of establishing business deals and partnerships in this today's world of business. This can be done by contracts. The contract types are decided by the parties involved in the business engagement. In ground reallity and matter of fact, the type of the contract used for the business engagement varies mostly depending on work type and industrial nature. The contract is nothing but it's an logical and elaborated understanding and agreement between two or more parties. One or more parties may provide products or services in return to something provided by other parties (client). The contract type plays the key role in relationship between the parties engaged in the business and the contract type determines the project risk [22].

Let' us see most widely used contract types in all engineering industries as well in software industry.

2.2.1 Fixed Price (Lump Sum). This is one of the simplest contract type. The terms are quite simple, straightforward and not so difficult to understand. The SP (service provider) agrees to provide a defined service for a decided or specific time period. The client gives word to pay a fixed amount for the service. This contract type may decide certain milestones for the deliveries and also KPIs (Key Performance Indicators). In addition, the software contractor may have his(r) an acceptable criteria defined and fixed for the milestones and the final delivery. The main significance of contract type is that the client knows the total fixed project cost before the project starts.

2.2.2 Incentive. An uncertainty in project cost is the main cause and reason of making this type of contract. The technological challenges gives effect on all effort and resources though there are accurate estimations in this contract. There are three cost factors in an Incentive contract; target price, target profit and the maximum cost. Both the parties should feel ease as this contract device targeted price overrun between both. The SP and client helping hand provides to minimise the risks in the business for both parties.

2.2.3 Retainer (Time and Material - T\&M). The beauty of this contract is two or more parties get together in the business engagement. All the parties are always in somewhat relax state as this is risk-free type contract. In this contract, the project time and material used are priced. The contractor has to only require to know the time and material for the project in order to make the payments. This contract has short and sweet delivery cycles, and separate estimates for each cycle are sent to the contractor. Once the contractor 
make the agreement and signs off the estimate with $d$ Statement of Work (SOW), the service provider can have green signal to start work. The T\&M contracts are nearly utilized for long-periodic and tenure business engagements. This is not possible other contracts.

2.2.4 Unit Price. In this contract, the entire project is divided into units. The each unit or milestone is defined. This contract type can be placed and introduced as one of the more flexible methods compared to fixed price contract. Usually, the contractor and client of the project decides on the cost estimates. Both the parties asks the bidders to make bond and bid of each element of the project. After making bond and bidding, depending on the bonded amounts and the qualifications of bidders. The entire project may be given to different providers or the same service provider. Different units may be assigned and allocated to different SPs. This contract is really becomes important when different project units or milestones require different specialists and expertise to complete the project.

2.2.5 Cost Plus. The services provider is reimbursed for their labour, machinery and other costs. In this model, the agreed fee has to be paid by contractor to the service provider. The detailed schedule has to be provided by the service provider. The resource allocation for the project is given by SP also. Periodically reporting to the contractor is must regarding all the costing in the budget of project. The payments may be paid by the contractor at a certain frequency (such as monthly, quarterly) or by the end of milestones.

2.2.6 Percentage of Construction Fee. This type of contracts are especially utilised used for engineering projects. Based on the resources and material required, the cost for the construction is estimated. Then, the client pays a percentage of the cost of the project as the fee for the service provider after the agreement. As an example, take the scenario of construction of home. Assume, the estimate comes up to Rs.4,00,000/-. When this project is contracted to a service provider, the client may agree to pay $25 \mathrm{PC}$ of the total cost as the construction fee which comes up to Rs. 1,00,000/-.

\subsection{The Schedule management}

Though, our approach is considering the direct cost, we emphasise on the adjustment of cost budgeting which is the combination of both direct and indirect costs. Ultimately, the cost of the complete project is combinations of the factors related to these both costs. We want to give free hand to PM for getting correct contingency plan for cost and time for different schedule plans with different contracts. ACWP is originally considered as direct cost which is related to labour cost only. But, some companies may take the AC as the combination of the both the direct and indirect costs. We refer the latter one AC for proposed approach so that we can have different schedule types in the hand of PM depending upon how the PM decides to cut the angles of features (including time and cost). The Schedule management in SPM (Software project Management [50]) consists of criteria and the activities, used for development of software project, are based on the needs of the project. The schedule management consists of schedule activities, its analysis, technique (making time span short), schedule control (process). Not only, these factors but also, SPI (Schedule Performance Index), SV (Schedule Variance) give idea how to control the project with various schedule by mean of DUR (duration of project).

$$
\begin{gathered}
S P I=\frac{E V}{P V} \\
S V=E V-P V
\end{gathered}
$$

Table 3. INPUT:Task Properties [9].

\begin{tabular}{|c|c|c|c|c|}
\hline $\begin{array}{c}\text { Task } \\
\text { id }\end{array}$ & $\begin{array}{c}\text { Efforts } \\
(\mathrm{PM})\end{array}$ & $\begin{array}{c}\text { CD } \\
\text { Months }\end{array}$ & $\begin{array}{c}\text { CPD } \\
\text { Months }\end{array}$ & $\begin{array}{c}\text { Required } \\
\text { skills }\end{array}$ \\
\hline 0 & 10 & 6 & 9.6 & 1,2 \\
\hline 1 & 15 & 7 & 11.2 & 3,4 \\
\hline 2 & 20 & 8 & 12.8 & 4 \\
\hline 3 & 10 & 6 & 9.6 & 1,3 \\
\hline 4 & 15 & 7 & 11.2 & $2,3,4$ \\
\hline 5 & 15 & 7 & 11.2 & 1,3 \\
\hline 6 & 10 & 6 & 9.6 & 2,4 \\
\hline 7 & 10 & 6 & 9.6 & 1,5 \\
\hline 8 & 20 & 8 & 12.8 & 3,4 \\
\hline 9 & 20 & 8 & 12.8 & 3,5 \\
\hline 10 & 10 & 6 & 9.6 & 1,2 \\
\hline 11 & 15 & 7 & 11.2 & 3,5 \\
\hline 12 & 20 & 8 & 12.8 & 4,5 \\
\hline 13 & 25 & 8.5 & 13.6 & 2,5 \\
\hline 14 & 15 & 7 & 11.2 & 4,5 \\
\hline 15 & 10 & 6 & 9.6 & 2,4 \\
\hline 16 & 15 & 7 & 11.2 & 2,5 \\
\hline 17 & 10 & 6 & 9.6 & 2,3 \\
\hline
\end{tabular}

Table 4. INPUT:Employee property table [9].

\begin{tabular}{|c|c|c|c|c|}
\hline $\begin{array}{c}\text { Employee } \\
\text { id }\end{array}$ & Salary & Month & Overload & skills \\
\hline 1 & 5000 & 1 & 1.1 & 3,4 \\
\hline 2 & 4000 & 1 & 1.15 & 1,3 \\
\hline 3 & 3000 & 1 & 1.2 & 2 \\
\hline 4 & 5000 & 1 & 1.15 & $3,4,5$ \\
\hline 5 & 3000 & 1 & 1.15 & 1 \\
\hline 6 & 6000 & 1 & 1.15 & $2,3,4$ \\
\hline 7 & 6000 & 1 & 1.15 & 2,3 \\
\hline 8 & 5000 & 1 & 1.15 & $2,3,4$ \\
\hline 9 & 8000 & 1 & 1.15 & 2,3 \\
\hline 10 & 9000 & 1 & 1.15 & $1,2,5$ \\
\hline
\end{tabular}

where, EV is earned value and PV is planned value. The SPI or SV are used to see the performance of the project schedule. If SPI $>1$ and $\mathrm{SV}>0$ then it indicates, project is within controlled plan in real development SPLC time [32]. This is dependent upon baseline, the approved plan for project, WBS (work breakdown structure), it's components and target schedules. SPI and SV can used for calculation of fitness for getting target schedule, also [35].

\subsection{Baseline}

The baseline includes cost, DUR and scope of the project. Various schedules are inputs to the baseline of software projects. The types of schedule can be used and adapted in the baseline depending upon the scope baseline, scope change, scope control, scope creep. All above scope management activities give opportunity to PM (project manager) to adopt the schedule type accordingly. Contingency allowances give also space to PM to adjust in fullback position by using the money or time and both for coming up from the overrun. Contingency plan gives 10PC on DUR or cost as the contingency plan dependent on CB (cost baseline).

\subsection{Software Cost and budgeting}

Software cost estimation and budgeting [7] is uncertain business thats why, we require requirement elicitation, undivided and impor- 
tant part of RE (Requirement Engineering). This uncertainty relates with cost growth and negative CPI (cost performance index [35]), are main reasons of inaccuracy in the cost estimation and schedule cost. The software requirement engineering is the important part for getting the requirement analysis in clear manner so that, we can improve budgeting by requirement specificity for keeping the accuracy in estimating, efficiency of costing i.e. with keeping CPI in better range. Another uncertainty, in the software cost estimation is making under-estimation of software size (KLOC). The study of Hihn and Habib shows that best estimation is mean estimation of underlying effort. BAC (Budget at Completion) is sum of the estimated cost of every tasks or work to setup cost baseline as cost baseline is the part of main baseline. CEVP (cost estimate validation process) is another way of making the cost/budget fixing and is the part of CMP (Cost Management Plan), in turn; CMP is the part of project management plan. This plan and process can be adjusted with each CPI of every phases during the project development in SDLC. Just like SPI [32], CPI gives measurement for cost efficiency and is defined as

$$
C P I=\frac{E V}{A C}
$$

where $\mathrm{EV}$ and $\mathrm{AC}$ are in target scheduling. $\mathrm{AC}$ is expenditure cost also. Keeping various EV value, we can have different schedules. $\mathrm{PM}$ can see the contingency plan where schedule cost and DUR will affect on CPI as actual cost includes various contract features and method. These contracts are CPF (cost-plus-fee) contract, CPFF (cost-plus-fixed-fee ) contract, CPIF( Cost-plus-Incentivefee) contract. These reimbursable contracts are always the part of the cost budgeting. These types of contracts gives PM to move around in the schedule plan for making the trade-off between various schedule planes and schedule cost in baseline (original or first or basic plan) at construction (build) phase of SPLC. As SPI can be used for the fitness in target scheduling, CPI can be used in adjusting the indirect cost.

\subsection{About SCE and it's step [31] [43] [49] [7]}

Software cost estimation plays different role at different stages or phases of SPLC. Schedule plan or even baseline of SCE is dependent on different role at different stages or phases of SPLC . In early stage of SPLC, generally emphasize is given on design related part and later on it becomes the part of management i.e. scheduling.

- Schedule Cost Estimations may be done by an expert or team of specialists, analytical way of historical data, various models along with thumb rules.

- Some industries satisfies with PC effort estimation by phase wise. There is standard PC distribution of effort, can be taken from Uniform Software project management phase wise distribution table.

- This phase wise or WBS wise estimation may be done by taking parametric cost models or by mathematical relationships. This type of estimation has relations of parameters at various levels and stages of projects of similar or same type. These typical parameters are defined for specific type of projects. The relation between them gives mathematical model expressions for every phases.

- Historical analogy estimation methods are dependent on effort, phase wise cost of past projects which are completed successfully.

- Expert judgment is another way of doing estimation as the experts are related to their domain.

is Steps of Software cost estimation [6]

Software estimation consists of many tasks and functions to do in systematic steps. Elicitation of information, requirements, defining the work elements, software size estimation, estimation of effort, scheduling of effort, cost estimation, determination of risk effects, validation of estimation or budget by analogy, reconstructing the budget and schedule, reviewing and approval of estimates, monitoring the cost expenditure Vs budget allocated maintain the linearity in it [7] [30]. These are all the steps in software estimation which are the part of software schedule plan it self. Among these, we are concentrating on scheduling plan which is the prime work of software manager, estimator, cognizant engineer.

2.6.1 Scheduling the Effort. This is the main part of the project cost estimation where we have to calculate the time required to complete the project according to WBS. Every WBS has work element with proposed time requirement. Letting of $10 \mathrm{PC}$ of schedule time is generally allowed as margin as it is regular practice. One month is given margin for one year schedule. Determining the order or sequence is the next step. The dependencies between activities and WBS components are also in the drive. The project schedule margin is considered to adjust the CT (Critical Time) and pessimistic duration in which, the CT is calculated by PDM (precedence diagramming method). CT is longest path in the activity network of schedule and can be taken as threshold for the completion of the project.

2.6.2 Calculating the Direct Cost (ACWP). This step has some sub steps. These are determination of cost procurements, cost proposed training period, determination of salary and skills proficiency. It is necessary to iterate the estimation several times by iterative methods. This estimation is cost value EV. $55 \mathrm{PC}$ of projects goes beyond the budget (EV) that to by over and above 90 PC (according to Remer [3]). 8 to 18 PC time should be given to developing the plan also, depending on technology used for project.

2.6.3 Validating the Estimate. The thumb rule tells to keep alternate estimation technique for comparison, these may be and are expert opinion estimation and model-based estimations. We can do comparison of our estimated cost with any other model. Generally, comparison parameters are size, effort, and cost of same type of projects. Resolving the differences, reviewing-refining the schedule and schedule plan are continuous process in SPLC and to be done as regular checkpoint. That's why we developed plans for not only for baseline also for target scheduling. Proposed model can be , also, used as target scheduling model.

2.6.4 Reconcile Estimates, Budget and Schedule . Cost of individual functions, precedence of it, inter communications are considered in this step. In real practice, one should reduce the functionality to reduce the function points in terns to get the tasks to accommodate in budget. Budget margin calculation is done as follows

$$
\begin{aligned}
\text { CostMargin } & =\frac{(P E V-C E V)}{P E V} . \\
\text { ScheduleMargin } & =\frac{C S H D-G R S H D}{C S H D}
\end{aligned}
$$

where $\mathrm{PEV}=$ proposed $\mathrm{EV}, \mathrm{CEV}=$ COCOMO-EV, CTT=COCOMO Threshold time, ET=estimated time. Above margin is used for minimizing the cost and time for calculating the fitness of individual schedule plan

\section{GA, GENEREPAIR AND GRGA [28] [25]}

GA is a generate-and-test population-based search stochastic method to solve the tough problems. The diagrammatically simple 


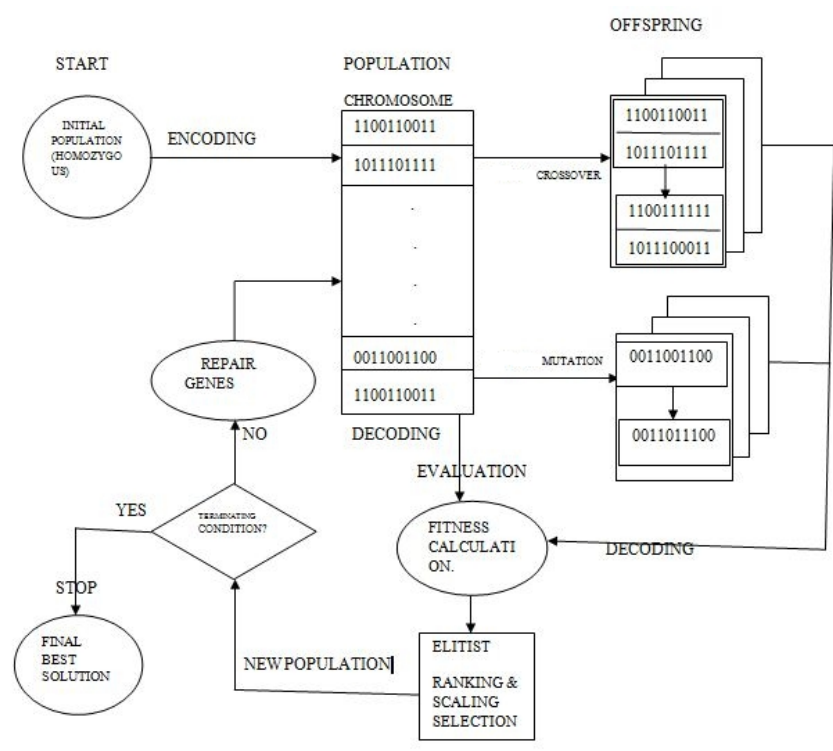

Fig. 1. Proposed GRGA Approach

GA can be shown as fig.3. The evaluation function does a heuristic estimation quality solution. The search process is completely taken in the hand by the variation and the selection operator.

\subsection{GA Components}

GA consists of representation i.e. representation and definition of individuals, evaluation function means fitness function or compound function, population, next generation individuals i.e. parent selection mechanism, variation operators (crossover and mutation), survivor selection mechanism (replacement) [37] [24].

3.1.1 Representation. Phenotypes are types forming possible solution within space of original problem. The encoded individuals in the GA evaluation are called genotypes. The representation is the mapping from set of the phenotypes onto a set of genotypes. Possible solutions consists of Candidate solution, phenotype and individual. This entire space is knows as phenotype space. Chromosome, and individual are the points in the genotype space. Single unit element in a chromosome is called gene. A gene value is called an allele. This representation is shown in fig.4.

3.1.2 Variation Operators. The role of variation operators ( crossover and mutation) is to create new generated individuals from past and old ones.

1. Mutation Operator : Mutation is a unary variation operator to apply on genotype. It produces the child or Offspring. Mutation is important operation which can guarantee about connected space.

2. Crossover Operator : Crossover or some time called recombination is a binary variation operator which merges information from two parent genotypes which leads and results into one or two offspring genotypes. Similarly to mutation, crossover is also stochastic operator. The random drawings are significance in this operation as choosing the parts of each parent, combining them and it's way and method depend on random drawings. The purpose of crossover is of mating two individuals which are different but having desirable features . This operation produces an offspring which combines both the features.
3.1.3 Parent Selection Mechanism. The role of parent or mating selection is to make comparison and distinguish among individuals based on their quality which allows the better individuals to become parents for forthcoming generation. The mating selection is based on probability and high quality individuals get a more chance than those of lower quality to become parents. Nevertheless, selection gives small but positive chance to low quality individuals, otherwise the complete search can get block and stuck in a local optimum

3.1.4 Survivor Selection Mechanism. As opposed to stochastic parent selection, survivor selection is always deterministic. e.g., ranking the unified multiset of parents and offspring which selects the quality and top segment (fitness biased).

3.1.5 Initialization. Initialization is generally put in simple form in most GA applications. This steps requirement depends on application at hand as it may be result in extra computational effort or not so much also.

3.1.6 Population. As population is a genotype multiset, the function of the population is to keep possible solutions. Usually in all GAs applications, the size of population is kept constant.

3.1.7 Termination Condition. As GA is stochastic and mostly, there are seldom guarantees to get an optimum way and solution. The following are the commonly used terminations conditions and These are as :

1. if the maximum letting CPU times elapses.

2. if The fitness evaluations reaches a given limit.

3. if the fitness improvement get stuck up at same value and keep itself under a threshold value,for a given period of time.

4. the population diversity goes down and drops under a given limit and threshold.

The traditional and simple GA is given as below in algorithm and also diagrammatical representation in fig.3.

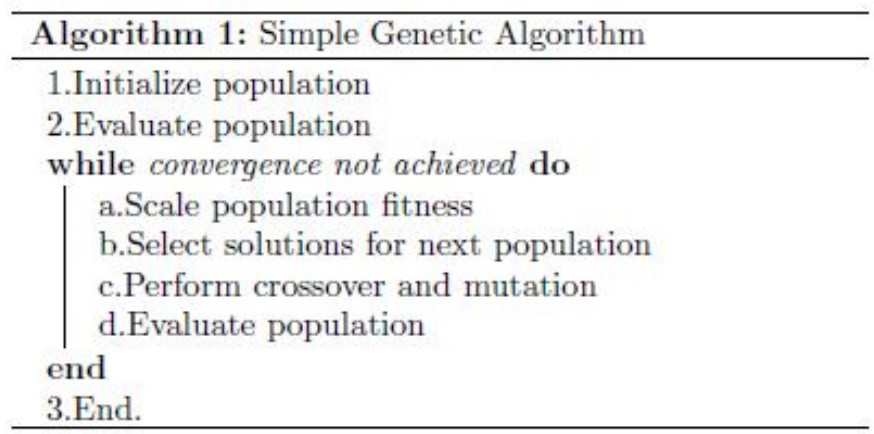

Fig. 2. Genetic Algorithm.

\section{ASSUMPTIONS, BUILDING BLOCKS AND PROBLEM DEFINITION FOR PROPOSED GRGA APPROACH}

The GRGA operates in traditional genetic algorithms manner, and is simply summarized as follows and shown in fig.1:

Our problem can be divided into two parts : Optimization and Permutation. A SGA (Holland, 1975) can effectively handle the first one i.e. optimisation. The upheld task of only allowing valid 


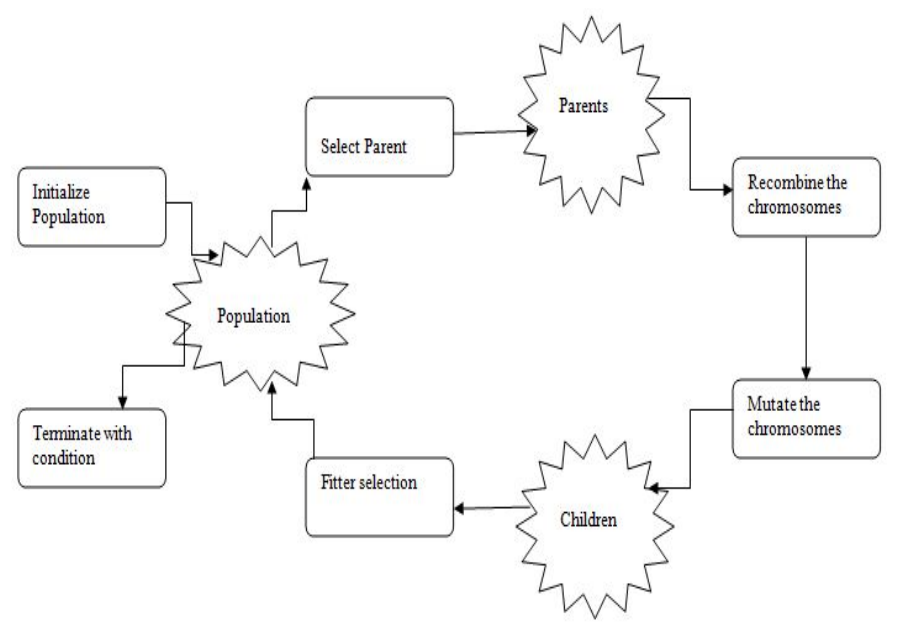

Fig. 3. Diagrammatical presentation of Simple GA.

individuals in the population lies solely with the GeneRepair operator.

As, proposed GRGA approach [24] gives the direction to cognizant engineers and estimators for differentiation and deviation between two estimation models. Thats why, we proposed different types of schedule with various constraints and conditions which gives alternative ways to show to the top management in different scenarios. Schedule types are based on single variable constraints, multiple variable constraints, non-constraint objective function type optimization techniques involved in genetic algorithm. Single variable constraint or single objective component function can be solved by other optimization techniques also. These techniques are linear programming, convex or concave optimization technique for two variable optimization. There are some cases or situations where we dont want to optimize anything. This type of feasibility problems only satisfies constraints to the model and not having objective. So the schedules are only dependent on soft and hard constraints . The example is problem solved for skill matching taken as hard constraint. Another type of objective function is multiple objective functions, are used to optimize number of different objectives simultaneously [39].

For optimization problem, variables are essential than the constraints [40]. We considered also functional constraints and side constraints also. As, the functional constraints are behavioural constraints which gives boundaries to the performance of a system rather than using the behavioural constraints, we used side constraints which is applicable for schedule design variables e.g. availability of a skilled staff.

Suppose $\exists$ A, B,C the sets of real or integer valued data for respective and particular range of the variables. As we have taken set $\mathrm{T}$ $=$ number of task, $\mathrm{E}=$ number of employees, $\mathrm{S}_{k}=$ set of skills. All these sets are to be intersected and added (union) by taking genetic algorithm into consideration, for representation and optimization with respect to the constraints applied on these elements of the set to get another set GRSHD of sub sets \{ GRSHD1, GRSHD2..., GRSHDn $\}$ which are nothing but number of feasible schedules.

\subsection{Assumptions and Concepts of Software schedule estimation [7] [8]}

The team of programmers, testers, coders, S/W project managers are used as an employees for assigning software engineering tasks [49] [21]. Since they are from every process groups nearly, we considered the some aspects of software engineering project management and software engineering principles. The well-known COCOMO model is utilized to get duration of the project by

$$
C D=2.5 \times \text { efforts }^{3.8}
$$

for making comparison between COCOMO-EV and proposed approach GRGA-EV. AC is taken as schedule cost which strictly related to working labour cost calculated after completion of WBS or activity and it is on going process during the project. For complete budgeting, we can have an EAC (Estimate at Completion) .

$$
E A C=A C+E T C
$$

, where ETC is extra and additional cost needed to complete the project. The ETC gives us to cover up an overrun. AC is used for calculating next target schedule. The estimated amount of time needed to accomplish every task is provided according to the basic COCOMO model. The input (Table 3 and Table 4) to our engineering schedule is properties of FTS(Full time software professional), activity or task, and PDM (precedence diagramming technique). The employee properties are employee id, employee skill, salary per month, their capacity in PC (percentage). The minimum capacity of employee is 152 hours per month or 22 days per week. Effort is taken as PM (Person months) for this paper. (The different companies assumes and takes differently this measurement as MM( man-months), SH(staff-hours), SW(staff-weeks) and $\mathrm{SD}$ (staff-days)). This means every employee gives 152 hours effort in person per month all together for one to many tasks. The maximum capacity of employee is multiple factor from 1 to 1.5 of standard effort according to the experience and skilled employee efficiency.

\subsection{Problem definition and formulation [12] [41] [34]}

A project schedule is an assignment of the tasks according to the 4Ps at particular duration by considering all the constraints of the project to get the optimal solution with optimum cost and time. Each task requires a set of skills and effort.

- Let $\mathrm{T}$ be a set of tasks, $\mathrm{T}=\left\{\mathrm{T}_{i}, \mathrm{i}=0, \ldots, \mathrm{n}-1\right\}$ where $\mathrm{n}$ is the number of tasks,

- Let $\mathrm{E}$ be a set of employees, $\mathrm{E}=\left\{\mathrm{E}_{j}, \mathrm{j}=0, \ldots, \mathrm{e}-1\right\}$ where e is number employees,

- Let $\mathrm{S}$ be a set of skills, $\mathrm{S}=\left\{\mathrm{S}_{k}, \mathrm{k}=0, \ldots, \mathrm{m}-1\right\}$, where $\mathrm{m}$ is the total number of skills.

- Let ES be a set of skill of employees,

$\mathrm{ES}=\left\{\mathrm{ES}_{i}, \mathrm{i}=0, \ldots ., \mathrm{m}-1\right\}$ where $\mathrm{m}$ is the number of skills and

- EF be the effort required for the tasks in T,

$\mathrm{EF}=\left\{\mathrm{EF}_{T_{i}}, \mathrm{j}=0, \ldots, \mathrm{n}-1\right\}$ where $\mathrm{EF}_{T_{i}}$ is the effort required for task $\mathrm{T}_{i}$.

- The skills required by tasks are represented by an $\mathrm{n} \times \mathrm{m}$ sized task skill matrix i.e TS,

where $\mathrm{TS}=\left\{\mathrm{TS}_{i k}, \mathrm{i}=0, \ldots ., \mathrm{n}-1, \mathrm{k}=0, \ldots, \mathrm{m}-1\right\}$

Each elements $\mathrm{TS}_{i k}$ of task skill matrix $\mathrm{S}$ is either 0 or 1 ,

depending on whether task $\mathrm{T}_{i}$ requires skill $\mathrm{S}_{j}$ as 


$$
T S_{i j}=\left\{\begin{array}{cc}
1 & \text { if } \text { Task } T_{i} \text { requires skill } S_{j} . \\
0 & \text { Otherwise. }
\end{array}\right.
$$

- The employee skills are represented by an e $\times$ m sized task skill matrix i.e ES,

where $\mathrm{ES}=\left\{\mathrm{ES}_{j k}, \mathrm{j}=0, \ldots, \mathrm{n}-1, \mathrm{k}=0, \ldots, \mathrm{m}-1\right\}$

Each elements $\mathrm{ES}_{j k}$ of employee skill matrix $\mathrm{S}$ is either 0 or 1 ,

depending on whether task $\mathrm{E}_{j}$ has $\mathrm{S}_{k}$ as

$$
E S_{j k}=\left\{\begin{array}{cc}
1 & \text { if Employee } E_{j} \text { has skill } S_{k} . \\
0 & \text { Otherwise. }
\end{array}\right.
$$

The dependence [41] between the tasks is given by task dependency matrix (TD) of size of $\mathrm{n} \times \mathrm{n}$. Its elements are given as,

$$
T D_{i k}=\left\{\begin{array}{cc}
1 & \text { if Task } T_{i} \text { depends upon task } T_{k} . \\
0 & \text { Otherwise. }
\end{array}\right.
$$

Finally, GRSHD is a $\mathrm{n} \times \mathrm{e}$ sized task assignment matrix of duration (in months) assigned to each employee on various tasks. The duration may be in years, months, quarters or weeks. TD matrix is obtained from task precedence graph (TPG). The Task Precedence Graph shows the precedence relation between the tasks, is an acyclic Graph, G(T,EG) where the T represents the set of all task nodes included in the project and EG is the set of edges between dependent tasks [20] [41].

\subsection{Chromosome structure}

The GRGA uses string type of chromosome. The combination 1,2, $3,4,5$ is the string which suggests us dedication of employee to the tasks. It is $0,0.25,0.5,0.75,1.0$ which determines the dedications in the solutions. Some example in the tables give the values $0,0.5$, $1.0,1.5,2.0$. The capacity of employee is taken as double. In other words, devotion granularity is increased from week to 15 days devotion with respect to months. In the first devotion, if a month is assumed as capacity then $0.25 \%$ devotion is a week and in other

\begin{tabular}{|c|c|c|c|c|c|c|c|c|c|}
\hline & Gene Value & \multirow[b]{2}{*}{ to } & \multirow[b]{2}{*}{$\mathrm{T} 1$} & \multicolumn{5}{|c|}{ String representation of chromos } & \multirow[t]{2}{*}{ Task } \\
\hline & & & & $T_{2}$ & T3 & TO & T1 T2 & $\mathrm{T} 3 \longleftarrow$ & \\
\hline & & 1 & 3 & 4 & 5 & 2 & 1 & Genotype & \\
\hline Employee & & Eo & & \multicolumn{5}{|c|}{ E1 } & \\
\hline & & 60 & 0.5 & 0.75 & 1 & 0.25 & 0 & Phenotype & \\
\hline & Schedule /So & olut: & ion $v$ & alue & & & & Dedication & 50.5 \\
\hline
\end{tabular}
example it comes out to be half of month ie. 15 days if capacity of employee is 2 months.

Fig. 4. Chromosomal representation in GRGA.

$$
\begin{gathered}
C D_{T_{i}}=2.5 \times \text { efforts } \\
C P D_{T_{i}}=C O P_{T_{i}} \times 1.6 \\
C L D_{T_{i}}=C O P_{T_{i}} \times 1.3 \\
C E D_{T_{i}}=\left(C D_{T_{i}}+C P D_{T_{i}}+C L D_{T_{i}}\right) / 3
\end{gathered}
$$

where, CED, CLD, CD, CPD are COCOMO Expected duration, COCOMO likely duration, COCOMO duration and COCOMO pessimistic duration respectively for $\mathrm{T}_{i}^{t h}$ task. This estimation formula gives importance to Likely estimation but proposed approach take the CPD into consideration which gives free hand to the manager to get the managerial adjustment. It is as hard constraint to every schedule types which gives ultimate solution which is from the space of likely effort so that all the solutions should be clustered in the likely part of the effort hypothesis. All these data are used for construction of schedule with the goal of completion of all assigned tasks by different objectives. The objectives are given in next subsection in the form of schedule type. Many different factors results in very complex scheduling process. Following subsection illustrates and shows schedule type number, schedule type's objectives, hard and soft constraints.

\subsection{Solution constraints}

Combinatorial problems like our SPSP place constraints on solutions [21] [23]. Solutions are only valid when all Hard constraints in the problem are satisfied in the solution. Thus, we used a fixed-length 2D-chromosome to represent our different schedules as shown in fig.4. Furthermore, a solution is valid when hard constraints defined in the schedule types are fulfilled by once in the solution. These constraints play role for application of the GeneRepair operator. The crossover can cause a violation of the validity constraint such as ordering the tasks in the schedule, by combining parent, which result in invalid individual. Similarly, mutation operators can also produce and generate invalid solutions. This happens when mutation randomly inserts a employee that already being given to other task in the solution.

In practice, GeneRepair examines each schedule in turn, force them to follow the following things in our problem.

1. Correct number of employees according to head count required for the tasks.

2. No overlapping of employees.

3. No violation of task duration to cross the limit of pessimistic duration.

These constraints do invite the GeneRepair operator, and identifies the above things in schedule.

\subsection{Gene-Repair}

GeneRepair replaces the overlapped employees and, less and large task duration tasks, iteratively, with valid employees and tasks duration retrieved from a master individual template. Following algorithm-2 shows the generepair operator inserted as last operations in loop. It also gives stoping criteria as schedule and cost margin.

Three different types of template can be used [24]:

1. Fixed template. This consist of a preset valid schedule (generated by SGA), and remained constant throughout the generations. 2. Parent Template. Selected the fitter and elitist parent, and used that as the master template. This template varies for every individual corrected.

3. Random Template. For each corrected and valid individual a new template is generated, within the limitation of constraints of the SPSP.

Following algorithms (Algorithm-1,2,3) explain about gene repairing technique used in this paper. Gene repairing using random generation adjusts and changes the allele according to the value required for task duration. The repairing gene takes place in both the techniques whenever the task duration value GROD of task $\mathrm{T}_{i}$ 


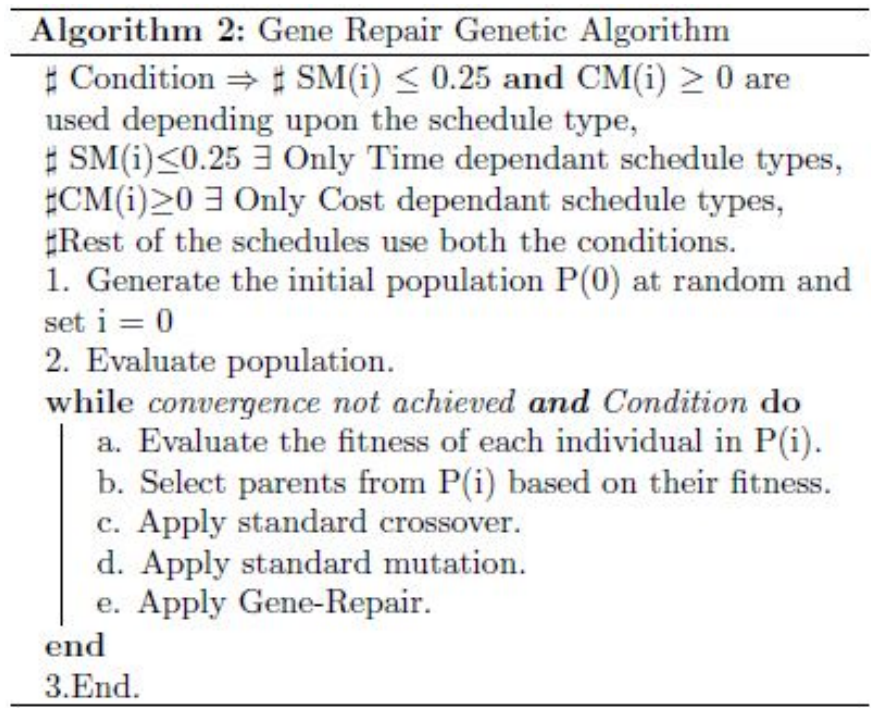

Fig. 5. Gene Repair Genetic Algorithm

above the $\mathrm{CPD}_{T_{i}}$ and below the $\mathrm{CD}_{T_{i}}$.

For first technique, if the $\mathrm{GROD}_{T_{i}}$ is higher than $\mathrm{CPD}_{T_{i}}$ then value of some alleles are changed to some lower value at random from 1 to 4 . The alleles which are in between 5 to 8 , are changed to 1 to 4 randomly and viceversa for value less than $\mathrm{CD}_{T_{i}}$ (Shown in Algorithm-3).

In second technique (Algorithm-4), the allele (value of gene) is changed according to the allele of corrective parent template. An algorithm- 4 explains the scenario where the allele is changed to $C P_{T_{i} E_{j}}$ if it is less than and greater than $C P_{T_{i} E_{j}}$ then and then only if task duration value GROD of task $\mathrm{T}_{i}$ below the $\mathrm{CD}_{T_{i}}$ and above the $\mathrm{CPD}_{T_{i}}$, respectively. $\mathrm{ES}_{T_{i}} \in \mathrm{TS}_{T_{i}}$ is applied to schedule type-7 and type- 8 only as it requires skill matching otherwise algorithm is without the condition of skill matching is used.

\section{STUDY OF SCHEDULE TYPES}

\subsection{Implementation [37] [10] [9]}

The code is written in JAVA using different GA classes in NET BEAN environment. The input parameters from input tables (Mentioned as INPUT in figs and tables )are taken as input to our Java code. The size of the population (number of different sets of parameter values considered for different schedule) is a user-defined variable. The default is 50 . We get the various outputs which has been in the output tables and figures.

The initial population of chromosomes uses randomly-generated values for all the parameters (although optionally one can include an initial guess as one of the chromosomes). Each chromosome in the population is created by taking each input parameter value, and normalising it to a number between 0 and 1 with respect to its fitting range. Parameters cannot leave their fitting range. The chromosome is then simply the list of these values, and the population the list of all the chromosomes. The population is regularly stored to disk during a calculation, and can also be

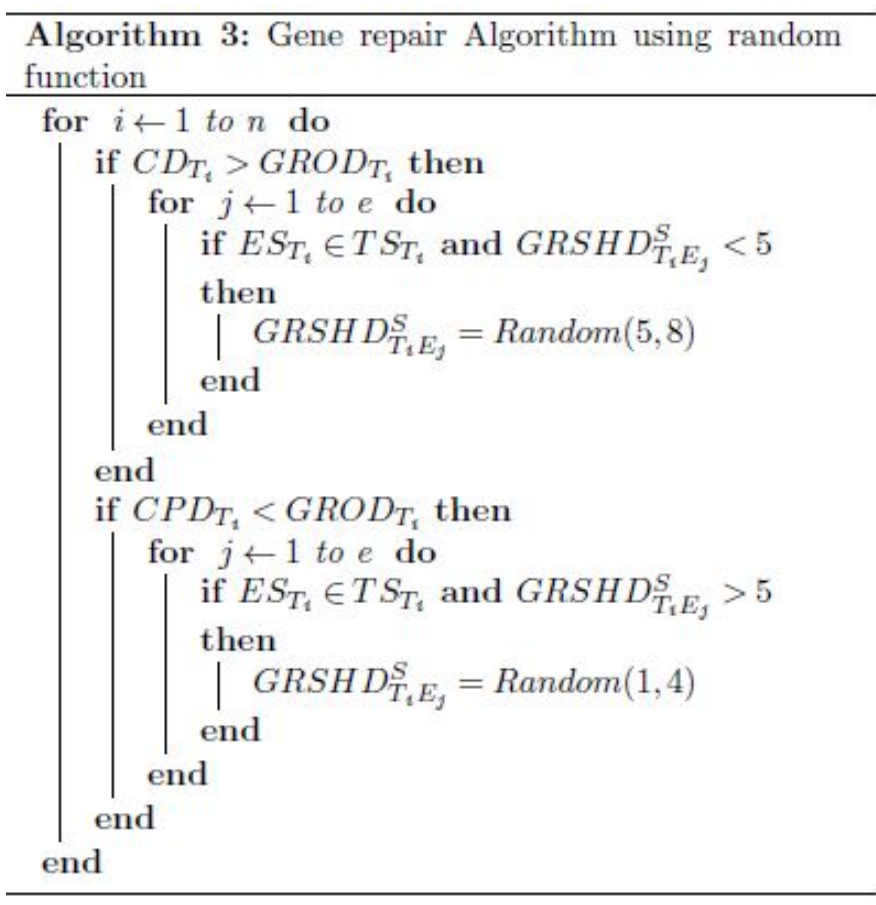

Fig. 6. Gene repair Algorithm using random function

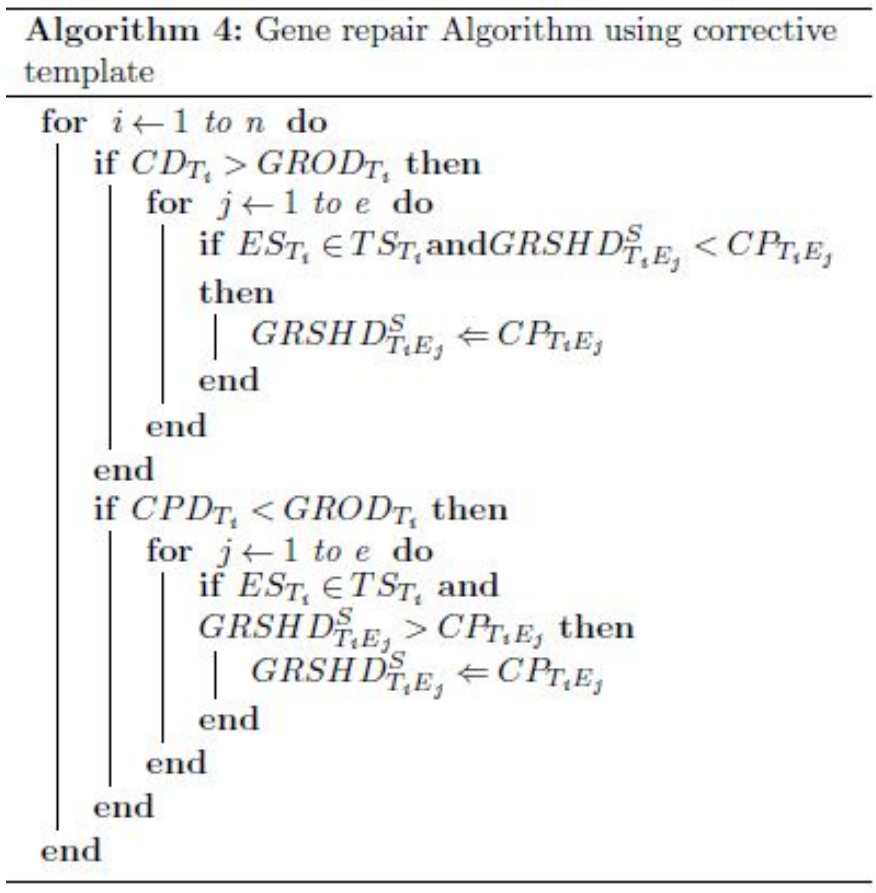

Fig. 7. Gene repair Algorithm using corrective template 
written on demand to facilitate restarting a run.

5.1.1 Evaluation of fitness. Genetic algorithms are normally maximising functions, so the fitness defined for each set of parameter values is calculated as

$$
N D_{s}=\frac{G R D_{s}}{\max _{i=1: S P} G R D_{i}}
$$

The above is the example given for only schedule type-2 but, the fitness formula changes as per the objective components change. Each time the fitness of a chromosome is better than the current notified by user and the parameter values are stored.

5.1.2 Selection of parents. The probability of a chromosome being selected as a parent for breeding purposes is linearly proportional to its rank by fitness as a fraction of the sum of the normalized objective components so better fitting parameters are more likely to be represented in the next generation. Selection is done by elitism method.

5.1.3 Breeding. Breeding,i.e.crossover is not always performed when creating children from selected parents a parameter $\mathrm{P}_{c}$ is defined which gives the breeding probability (default 0.85 ). If the parents are not bred, the children created are clones of the parents. Otherwise breeding occurs by taking the chromosome of each parent, listing the values as a single long string of numbers, choosing randomly a crossing point, and swapping all the digits after the crossing point from one chromosome to the other by crossover types.

5.1.4 Mutation. Each digit in each parameter value of the child chromosome is then assessed for mutation. The probability of mutation i.e. $\mathrm{P}_{m}$ is initially set to 0.01 , but varies during the routine within the range between 0.01 and 0.05 , increasing as the same fitness fitter chromosomes increases (and vice versa). If a digit is mutated, it is replaced with a random number (0-7).

5.1.5 Creation of new population. The above process is repeated as many times as necessary to create a new population of chromosomes the same size as the initial population. With the elite option set (default) the fittest chromosome (best set of parameter values) is always preserved from one generation to the next, ensuring that the best fit never becomes worse (although worse fits are obviously still considered in each generation, and contribute to the exploration of parameter space). The new population then replaces the old, and a new generation begins.

\subsection{Schedule Types and Discussions [37] [10] [9]}

The final output of GA schedule is pool of chromosomes. Although we have got same fitness chromosomes, there are various genotypes individuals available for selection for the project management.The project used in schedule is of 18 tasks, 10 employees, with the 5 skills. TT (Total Time) is considered as total efforts of the project is dependant on TT.

Following schedule types with their objectives and constraints are considered.

-Schedule Type 2(Only Total Time is considered).

-Schedule Type 2 (Hard Constraint:Task Completion,objective:minimum CT ).

-Schedule Type 2(Objective:Minimum CT).

-Schedule Type 2 (Objective:Minimum GRD).

-Schedule Type 3(Objective:Minimum simple Cost).
-Schedule Type 3(Objective:Minimum simple "Cost and Overtime as Penalty).

- Schedule Type 3 (Hard Constraint : Task completion).

- Schedule Type 4 (Objective: Minimum cost and Total time).

-Other schedule types objectives and constraints are explained in respective section. The other schedules constraints and objectives can be seen in the respective graphs also. The hard constraints and objectives are the combination of schedule type-2 and type-3 and stopping criteria for these schedules is fitness and combination of CPI and SPI conditions ( as mentioned in the algorithm). Some figures, tables shows and respective schedule subsection gives the soft and hard constraints required for every contract-schedule types.

Following equation is applicable for every schedule plan and it is hard constraint except schedule-3 Hard constraint is each and every employee must have at least one task assigned.

$$
\overbrace{G R G A D_{T_{i}}=\sum_{j=1}^{e} G R O D_{T_{i}, E_{j}} \geq 0}^{\text {For } i=1: n}
$$

5.2.1 Schedule type1. This is not realistic. Objective is no restriction on any triangular parameter and features. Schedule type 1 only, for evaluation cause where the output gives its maximum level of 180 as the optimal solution the result shows increase in the assignments of tasks as increase in the number of generations. This type of solution not feasible and not useful in real situation.

5.2.2 Fixed duration Schedule plan (schedule type 2) . FDS plan requires the schedule of having minimum time, there is no restriction on cost,working load. This type of schedule can be done when PMs put restriction of negative float(slack) due to strict timed schedule. The hard constraint is fixed duration. All FTS must have all the skills required for the projects. Total float is strictly followed as all the activities and tasks are put on ASAP category. All PMP groups (IPG, PPG, EPG, CPG, MPG, CLPG) and their concerned employees included in this schedule type.

$$
T F=P F+N F+F F
$$

where TF, PF, NF, FF are the Total float, positive float, negative float, free float respectively. Free float of every activity is kept, nearly, zero. Generally Crashing technique ( type of compression technique) is used. $\mathrm{CPF}$ (cost-plus fee) contract is suitable for this type of schedule.Nearly all activities are considered critical activity having zero float. Positive float is welcomed in this type of schedule. Each and every employee must have at least one task assigned. Hard Constraints are 1.

$$
\overbrace{G R D_{S}=\left(\sum_{i=1}^{n} \sum_{j=1}^{e} G R S H D_{T_{i}, E_{j}}\right)<C E D_{S}}^{\text {For } S=1: s}
$$

Here, CED is expected duration but already fixed by contract. Generally fixed duration is average duration calculated by an expert or COCOMO model.

2. $\mathrm{TF}_{S} \leq 10 \mathrm{PC}$ of $\mathrm{CED}_{S}$. 
3.

$$
\overbrace{G R O D_{T_{i}} \leq C D_{T_{i}}}^{\text {Hard Constraint }}
$$

This type gives the project manager minimum level project execution time with not having less constraints on project management it is a single variable objective function for optimization using genetic algorithm but the variable time is constant The schedule time or GRD is calculated additions of all the genes values as an Langrangs multiplier with devotion of employees, the objective of the function is to minimize time schedule only.

$$
\text { ScheduleMargin }=\frac{C C T_{s}-G R C T_{s}}{C C T_{s}}
$$

The proposed approach takes into account the task completion. The task completion definition is dependent on the COCOMO calculated duration time $\mathrm{CD}$. If contract is dependant on only on unit time i.e. milestones threshold time, it is up to the client and developers trade off which is DUR (duration) deadline for each milestone. Some may take CD as threshold or EDUR or CPD. It is totally dependant on the time required to complete the project. Generally this type of contract only considers the time not the cost which comes under the ST-2. This type of schedule is the type of fixed duration where every task duration is decided. It is also called as contractual time or unit time contract. Even though, second importance is given to the cost as minimum objective. The TPG is kept in consideration for this type of schedule.

Another fixed duration type where task completion is hard constraint and critical time is to be minimum. It is the combination of unit time and total time duration of the project. It is typical schedule where bonus or incentive in terms of leaves will be given. You can earn leave or take the cost in tern. This gives the free hands to the manager to select any person from team to fulfil the efforts of the activities, WBS component. Even though we are using TPG, the tasks can be completed by using parallel approach explicitly so that tasks can be completed below CED. BSD (Baseline start Date) and BFD (Baseline Finish Date) is strictly followed by only 10 PC margin.

Another type of schedule is schedule which takes into account only CT of project. This has got any hard constraint but objective is to have minimum CT. This type of schedule comes under only objective oriented optimisation.

Categorise of schedules are: Objective is minimum time

$$
F C_{s}=\frac{1}{G R D_{s}}
$$

Objective is minimum CT

$$
F C_{s}=\frac{1}{C T_{s}}
$$

Objective is minimum CT and Time penalty.

$$
F C_{s}=\frac{1}{C T_{s}}+\frac{1}{T P_{s}}
$$

Objective is TT and Time penalty.

$$
F C_{s}=\frac{1}{G R D_{s}}+\frac{1}{T P_{s}}
$$

Combination of above all:

$$
F C_{s}=T C \times\left(\frac{1}{C T_{s}}+\frac{1}{T P_{s}}\right)
$$

$$
T P_{s}=\left(G R G A D_{T_{i}}-C D_{T_{i}}\right) \times \eta
$$

where

$$
\eta=\frac{G R G A D_{T_{i}}}{C D_{T_{i}}}
$$

Where, $\eta$ is penalty rate to be decided by PM.

5.2.3 Fixed budget Schedule (Schedule type 3). Schedule must be of minimum cost, there is no restriction on working load. Contract overrun is not allowed. This type of schedule involves LumpSum contract where total price is fixed at the time of contract. CPFF(cost-plus-fixed-fee) may be the total fixed cost also. CPI is used for the re-planning of this type of schedule as it plays vital role in $\mathrm{AC}$ (to be considered during the project). CV plays important role in re-planning also. DC is calculated as lump-sum amount. Some companies fix the lump-sum amount after the calculation of DC. This ST3 types of schedules gives chance to the mainly IPG, PPG as BAC has to be corrected in PPG

$$
\left(D L C_{S}\right)=\left(\sum_{i=1}^{n} \sum_{j=1}^{e} G R S H D_{T_{i}, E_{j}} \times S a l_{E_{j}}\right)<\text { lump-sum }
$$

$$
L S A=D C_{p}=D L C_{S}+M C_{p}+E C_{p}+S C C_{p}
$$

where $\mathrm{p}$ is the project to be estimated, $\mathrm{S}=1: \mathrm{s}$, s: number of schedules.

Where DC $\Rightarrow$ Direct Cost, DLC $\Rightarrow$ Direct labour cost,MC $\Rightarrow$ Material Cost, $\mathrm{EC} \Rightarrow$ Equipment Cost, $\mathrm{SCC} \Rightarrow$ Sub-contract-cost. Salary of employee is excluding bonus, overtime, insurances and payroll taxes. Negative float activities are welcomed since as we can have time to do the project.

This schedule is of type which has minimum cost. This schedule type gives project manager as threshold in terms of money only. As project cost is hard constraint so that PM has to restrict him-self to complete the project within a proposed budget. The cost is dependent on the salary of the employee and ultimately and indirectly depends on the commitment of employee to the task. So, the schedule will be or may be showing the assignment to the employees which have the less salary. This type of schedule is useful only when we have more part time software professionals. Contract basis type of schedule comes under this category. We have two types schedule where the schedules can take two path. One is simple and only cost type schedule and second is genetic repaired cost type. First one tends to take the total cost into consideration and second one try to make to use as many as employees in the schedule. The second type schedule tries to complete the task as per proposed definition. First one takes the employee as many which has got the less salary but it happens partially in the second type. CPI is used as stopping criteria for the GRGA for both schedules.

$$
F C_{s}=\frac{1}{D L C_{s}}
$$

Above equation is for the simple cost schedule where only the salary of the employee is considered. It is the only single component object oriented optimisation problem where the objective is minimum cost. The schedule give more task to the less salary employee. This type of schedule is useful when there are professionals who work on contract basis. Total contractual salary can be fixed by any type of estimation. The proposed approach calculate two types of $\mathrm{DLC}_{s}$. First one is using simple GA and second one 
is using GRGA approach. Both types graphs for their fitness and cost verses generation has been shown in the figures it selves. The cost of using GA comes less than the GRGA as SGA does not take into account availability and task completion as per proposed approach definition. Hence, the value GRGA cost comes more as it calculates the cost after making GR individuals in GA. The task completion for SGA ( as per Carl Chang (2001)) is 'Each task must be assigned at least one employee'. Our definition considers COCOMO model's CD as mentioned earlier. GR is dependent on the $\mathrm{CD}$, that's why GRGA cost is more than SGA. 1 .

$$
F C_{s}=\frac{1}{D L C_{s}}+\frac{1}{O T_{s}}
$$

Above equation is optimisation of cost and over time as penalty. So the schedule will give the penalties if employees take the more time than the time required to complete the task. It means i.e. $\mathrm{CPD}_{T_{i}} \geq \mathrm{GRGAD}_{T_{i}} \geq \mathrm{CD}_{T_{i}}$. In other words, penalty will be given to the team. ( Here, OT is not the cost which gives more money while employee works more than his(r) capacity.)

$$
O T_{s}=\left(G R G A D_{T_{i}}-C D_{T_{i}}\right) \times \mu
$$

$\mu$ is the rate of penalty and

$$
\mu=\frac{G R G A D_{T_{i}}}{C D_{T_{i}}} \times 1.5 .
$$

2.

$$
F C_{s}=\frac{1}{C T D L C_{s}}
$$

where, $\mathrm{CTDLC}_{s}=\mathrm{TSM} \times \mathrm{GRCT}_{s}$ and TSM is team salary per month. $\mathrm{GRCT}_{s}$ is the critical time obtained by proposed method. The above equation shows the fitness is dependant on the total salary. The total salary of the employee may be constant or may be varied according to the performance of the employee or employees or team. The above schedule is dependant on critical time of the project. Ultimately, critical path is important and, decider of project cost. Typical tasks out of all tasks are given importance to complete the tasks in time. The task or the concurrent tasks should be completed within the limitations of CPM. This type of contract is dependant on the quality of the team and EMF of the team. As EMF for the team varies from $0.86 \mathrm{PC}$ to $1.6 \mathrm{PC}$, the more money will be given to the less EMF team as this team contains quality of professionals. Some time, this type of contact may be dependant on the personal EMF factors defined by COCOMO-II. 3.

$$
F C_{s}=T C \times \frac{1}{C T D L C_{s}}
$$

Another type of schedule gives importance to the time completion in terms of validation. Here, TC, Task Completed, is number of tasks satisfies the task completion criteria in GRGA. More weight will be given to the schedule as many as task satisfies this constraint. The objective is minimise the CTDLC.

$$
F C_{s}=T C \times\left(\frac{1}{C T D L C_{s}}+\frac{1}{O T_{s}}\right)
$$

The above equation shows the minimisation of two objectives and satisfying TC as soft constraints. The stopping criteria of GRGA for all above schedule is fitness and CPI condition (mentioned in algorithm).
5.2.4 Fixed time-cost Based Plan (T\&M type/Schedule type 4). Schedule and plan must be of minimum time and cost time. There is no restriction on working load. This type of schedule can be done when PM managers put restriction on negative floats not so strictly. All FTS must have all the skills required for the projects. Total float is not so strictly followed as all the activities and tasks are put on ASAP and ALAP category. The optimisation of all floating is done, ultimately tried to produce well floated schedule. CPIF(cost-plus-incentive-fee) contract is suitable for this type of schedule as this schedule is used for the comparison purpose with the others.Nearly all types of activities are considered. Critical activity is used for calculation. This type of schedule involves BAC and CAC costs. SPI is used for the re-planning of this type of schedule as it involves SC during the project. SV plays important role in re-planning also. IPG do the tasks of initialisation and RE as PPG plan for the schedule but other group of EPG, CPG, MPG, CLPG professionals can be used to involve in the project groups to achieve the objective of this type of schedule

$$
B A C_{p}=D C_{p}+I D C_{p}
$$

$$
I D C_{p}=\text { AdminCost }_{p}+\text { overhead }_{p}+\text { Generalcost }_{p}
$$

where $\mathrm{p}$ is the project to be estimated. This type schedule shows optimization of time and cost. These two variables are in the same directions of the hypothesis where we should have minimum time and minimum cost. Whenever there is time limitation for the software related to the size of team and its productivity.

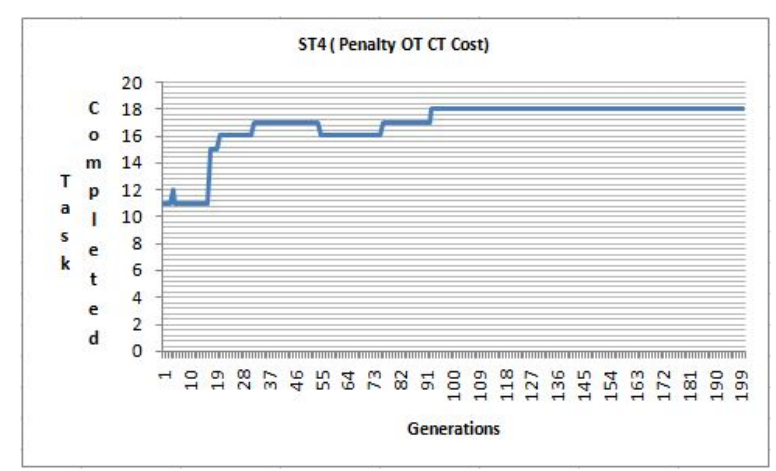

Fig. 8. Task Completion Vs Generation for Schedule Type 4(Objective: Minimum cost and Total time)

The formula in Equ.36 gives the most optimistic value of duration of every task but also that the duration of all the tasks required to complete the project. Though software project management allows one month per year or one month budget per year as margin that fulfils principle of management but, it also conflicts the total duration of the project as relief in the scheduling problem.

5.2.5 Cost-plus-incentive-fee-contract plan( target schedule plan 5 ). Schedule must be of minimum time, cost,working load. Costing is related to incentives for the employees. The incentives are calculated on the basis of employees approach and other factors of efficiency. Hard Constraint is duration of each task must be within limit.

$$
C D_{T_{i}} \leq G R G A D_{T_{i}} \leq C P D_{T_{i}}
$$

Cost-plus-incentive-fee-contract schedule considers composite function as minimum cost, time, penalty and rewards. The hard 
constraint is GRGAD ${ }_{T i}$ of each task $\mathrm{T}_{i}$. Each Task duration must be in between CD and CPD. The cost estimator can get this type of schedule if he want to restrict each completion task duration within a range of optimistic to pessimistic Every task must be completed within these two closed boundaries. CEVP process is adapted for this type of schedule. The objective function is the combination of cost, time, penalty and rewards. The reward or incentive is given accordingly if the task is in between likely and pessimistic in terms of money with a one and half rate of salary.

It is the type of combinatory problem of variable and constant constraints, the schedule not only compromises time with the cost but also the individual task duration and employees salary. This type of schedule is a real schedule which considers the importance of the efforts calculated by the COCOMO model, the duration of this type of schedule gives a chance to reduce further time of duration by keeping optimistic task duration intact.

5.2.6 Unit prise/Cost Plus Schedule (Schedule type 6 ). This type of schedule can be done when PM managers want to do the study of optimistic, pessimistic and likely duration for effect on threshold parameters like cost drivers, time , quality factors, resource values. All FTS must have all the skills required for the projects. SPI is used for the re-planning of this type of schedule as it involves SC during the project. SV plays important role in replanning also. CAC is considered as cost of project. Total duration is hard constraint, here.

$$
G R D_{s} \leq C T D_{s}
$$

The schedule type 6 considers minimum cost. Hard constraint is TC of the schedule. TC must satisfies the task completion criteria. This schedule comes under multiple objectives and single constraint mathematical problem where project manager has to restrict total average budget and total time.

The project manager has got the free hand to change the employee according to the bud-get and compensation to the time duration. Our solution in table- 5 and table- 6 gives the near optimal schedule which compromises mini-mum total duration and minimum cost by restricting the project duration in a total optimal duration.

Above schedule type is dependant on the millstones and or each task. The millstones may be group of tasks to be performed as processes in one group. The SLDC is having analysis, design, implementation and testing phases, usually. Each phase may contain all the process groups mentioned depending on the requirement of the activity or umbrella activities in the project. Each process group is assigned to typical skilled employees which are dedicated to the specific and repeated type of work. Before starting of the project, all the milestones or targets are decided by WBS. The cost of the every unit millstone is decided which is put on the paper as per the table shown above. This cost is obligatory to the client to pay after the completion every unit part of the project.

This type of schedule is adapted when there is very small projects or the project which has not so high risk. In contrast to this also, some company gets the very large project and distribute the works in unit to the sub-companies and collect it after some millstones by making legal contract with others. The very large projects are also do this type of schedule where they want to distribute the risks into many domains and process groups. The advantage of the unit prise contract type of schedule is any one can from the huge numbers of team can understand the risks and communicate to the top management without hesitation and lowering the high risks to the low risks by distributing the risks in the process groups.

Making the tiny parts of the huge project gives less responsibilities to the group to whom the unit is assigned with threshold date. This type of schedules require more management works than the actual work. This type of contract gives overhead to the management and management activities as this huge projet work has not only follow the TPG, but it has to follow the employee dependency also.

5.2.7 Quality schedule plan (Long Term business Plan) and schedule type 7. Nearly, all the parameters are considered as it required quality project to be delivered to the customer. Contract is all the combinations of subcontracts and contracts till we studied. There is no compromise with equality so the cost mostly includes the quality assurance and quality control related costs, hence the skill matching is must, here.

$$
E S_{T_{i}} \in T S_{T_{i}}
$$

The schedule type 7 considers skill matching and all objectives. Overloading is soft constraint.

This schedule is very important schedule to get a quality oriented project as skilled employee is only assigned to the task by considering minimum salary and minimum time. These two variables are exactly opposite to the skill matching constraint as project manager doesnt have choice but to assign employee to the task considering strictly skill matching. This type of schedule surpasses minimum time and cost as skill matching restrictions increases the somewhat the cost and time in initial phase, but, after some duration we will get less time.actually. Fig.9 shows loading of employees in schedule type- 7 .

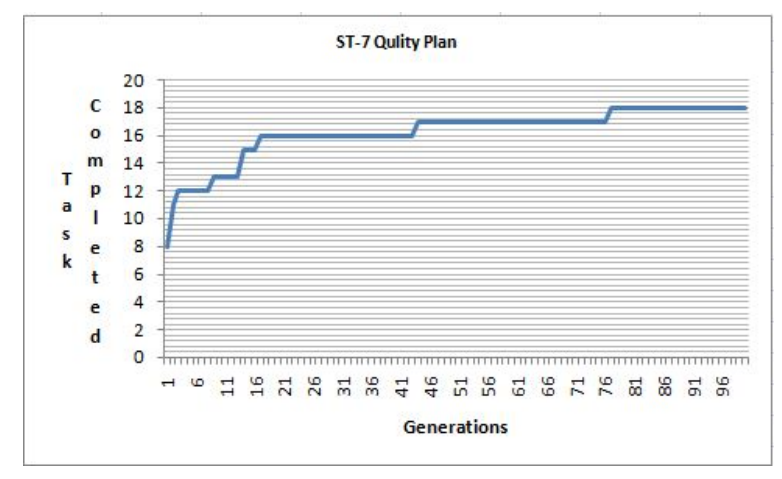

Fig. 10. Task completion Vs Generation for Schedule Type 7

The long term business plan in software industries require the skilled employee. This skilled employees are taken into consideration with respect to the EMF of programmer and team. Depending on this, salary is fixed or varies. The skilled employee gives the quality project as it takes less time with solid plinth. Dedicated skilled or unique skilled employee do the task very fast and give the better results in the project. This type of long term business plan needs to consider all the benefits to the employee. The management has to go through the various insurance policies, bonus and incentives as long term business plan certainly thinks about the relation of employees with employer to keep the momentum of the project in right path. The skilled employee with high salaried people or skilled employee with less salary employees are in this project and part of knowledge hub. The project is not only high risk and scope type but nevertheless it encompasses performance with 
Table 5. An Optimum Schedule type 6 ( Unit Prise )( objective:considering minimum cost, Hard constrained is Task completion).

\begin{tabular}{|l|l|l|l|l|l|l|l|l|l|}
\hline & T0 & T1 & T2 & T3 & T4 & T5 & T6 & T7 & T8 \\
\hline E0 & 1 & 0.5 & 1 & 0.75 & 0.75 & 0.5 & 0.5 & 0.75 & 0.5 \\
\hline E1 & 0.5 & 0.5 & 0.75 & 0.75 & 0.5 & 0.75 & 0.5 & 0.5 & 0.5 \\
\hline E2 & 0.5 & 1 & 1 & 1 & 1 & 1 & 0.75 & 0.75 & 1 \\
\hline E3 & 0.75 & 0.75 & 1 & 0.5 & 0.5 & 0.5 & 0.75 & 1 & 0.75 \\
\hline E4 & 0.5 & 1 & 0.5 & 0.5 & 0.5 & 0.75 & 0.5 & 0.5 & 1 \\
\hline E5 & 0.75 & 0.5 & 1 & 1 & 0.75 & 1 & 0.5 & 0.5 & 1 \\
\hline E6 & 0.75 & 0.75 & 0.75 & 0.75 & 1 & 0.75 & 0.5 & 0.5 & 1 \\
\hline E7 & 0.75 & 0.75 & 0.5 & 0.75 & 0.75 & 0.75 & 0.75 & 0.5 & 1 \\
\hline E8 & 0.5 & 0.5 & 0.75 & 0.5 & 1 & 0.75 & 0.75 & 0.75 & 0.5 \\
\hline E9 & 0.5 & 1 & 0.75 & 0.5 & 0.5 & 1 & 1 & 0.5 & 0.75 \\
\hline \hline GRGD & 6.5 & 7.25 & 8 & 7 & 7.25 & 7.75 & 6.5 & 6.25 & 8 \\
\hline \hline T9 & T10 & T11 & T12 & T13 & T14 & T15 & T16 & T17 & \\
\hline 1 & 0.5 & 0.75 & 1 & 1 & 1 & 0.5 & 1 & 0.5 & 13.5 \\
\hline 0.5 & 0.5 & 0.5 & 0.75 & 0.75 & 1 & 0.5 & 0.75 & 0.5 & 11 \\
\hline 1 & 0.5 & 0.5 & 0.75 & 0.75 & 0.75 & 0.75 & 0.5 & 0.5 & 14 \\
\hline 0.75 & 0.75 & 1 & 1 & 0.75 & 0.75 & 1 & 1 & 0.5 & 14 \\
\hline 0.75 & 0.5 & 0.75 & 1 & 0.5 & 1 & 1 & 1 & 1 & 13.25 \\
\hline 0.75 & 0.5 & 0.5 & 1 & 1 & 1 & 0.5 & 0.75 & 0.5 & 13.5 \\
\hline 1 & 0.75 & 0.75 & 1 & 1 & 0.5 & 1 & 0.5 & 1 & 14.25 \\
\hline 1 & 1 & 1 & 0.5 & 1 & 0.5 & 1 & 1 & 0.75 & 14.25 \\
\hline 0.75 & 0.5 & 1 & 0.5 & 1 & 0.75 & 1 & 0.75 & 0.75 & 13 \\
\hline 0.75 & 0.5 & 0.5 & 1 & 1 & 0.5 & 0.5 & 0.75 & 0.75 & 12.75 \\
\hline \hline 8.25 & 6 & 7.25 & 8.5 & 8.75 & 7.75 & 7.75 & 8 & 6.75 & 133.5 \\
\hline
\end{tabular}

Table 6. An Optimum Schedule type 6 ( Unit Prise )( objective:considering minimum cost, Hard constrained is Task completion).

\begin{tabular}{|c|c|c|c|c|c|c|c|c|c|}
\hline & T0 & $\mathrm{T} 1$ & $\mathrm{~T} 2$ & T3 & $\mathrm{T} 4$ & T5 & T6 & $\mathrm{T} 7$ & T8 \\
\hline E0 & 5000 & 2500 & 5000 & 3750 & 3750 & 2500 & 2500 & 3750 & 2500 \\
\hline E1 & 2000 & 2000 & 3000 & 3000 & 2000 & 3000 & 2000 & 2000 & 2000 \\
\hline E2 & 1500 & 3000 & 3000 & 3000 & 3000 & 3000 & 2250 & 2250 & 3000 \\
\hline E3 & 3750 & 3750 & 5000 & 2500 & 2500 & 2500 & 3750 & 5000 & 3750 \\
\hline $\mathrm{E} 4$ & 1500 & 3000 & 1500 & 1500 & 1500 & 2250 & 1500 & 1500 & 3000 \\
\hline E5 & 4500 & 3000 & 6000 & 6000 & 4500 & 6000 & 3000 & 3000 & 6000 \\
\hline E6 & 4500 & 4500 & 4500 & 4500 & 6000 & 4500 & 3000 & 3000 & 6000 \\
\hline E7 & 3750 & 3750 & 2500 & 3750 & 3750 & 3750 & 3750 & 2500 & 5000 \\
\hline E8 & 4000 & 4000 & 6000 & 4000 & 8000 & 6000 & 6000 & 6000 & 4000 \\
\hline E9 & 4500 & 9000 & 6750 & 4500 & 4500 & 9000 & 9000 & 4500 & 6750 \\
\hline UP & 35000 & 38500 & 43250 & 36500 & 39500 & 42500 & 36750 & 33500 & 42000 \\
\hline T9 & T10 & T11 & T12 & T13 & T14 & T15 & T16 & T17 & DC \\
\hline 5000 & 2500 & 3750 & 5000 & 5000 & 5000 & 2500 & 5000 & 2500 & 67500 \\
\hline 2000 & 2000 & 2000 & 3000 & 3000 & 4000 & 2000 & 3000 & 2000 & 44000 \\
\hline 3000 & 1500 & 1500 & 2250 & 2250 & 2250 & 2250 & 1500 & 1500 & 111500 \\
\hline 3750 & 3750 & 5000 & 5000 & 3750 & 3750 & 5000 & 5000 & 2500 & 70000 \\
\hline 2250 & 1500 & 2250 & 3000 & 1500 & 3000 & 3000 & 3000 & 3000 & 39750 \\
\hline 4500 & 3000 & 3000 & 6000 & 6000 & 6000 & 3000 & 4500 & 3000 & 109750 \\
\hline 6000 & 4500 & 4500 & 6000 & 6000 & 3000 & 6000 & 3000 & 6000 & 85500 \\
\hline 5000 & 5000 & 5000 & 2500 & 5000 & 2500 & 5000 & 5000 & 3750 & 71250 \\
\hline 6000 & 4000 & 8000 & 4000 & 8000 & 6000 & 8000 & 6000 & 6000 & 156750 \\
\hline 6750 & 4500 & 4500 & 9000 & 9000 & 4500 & 4500 & 6750 & 6750 & 114750 \\
\hline 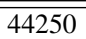 & 32250 & 39500 & 245750 & 449500 & 40000 & 441250 & 442750 & 37000 & 870750 \\
\hline
\end{tabular}

respect to the high profile employees in management and technical fields. The skilled employee can be classified as per the five process groups mentioned earlier to do the specific tasks in the team. Team can be given name as per the process groups also. The main part of the quality plan is to do the interaction between the process groups as per the PMBOOK. All group employees can assign to every phase of SLDC. The smooth interaction between the groups imparts the results in quality project. 
Table 7. An optimum Schedule 7: skill matching, all above objectives and constraints with overloading as soft constraint.

\begin{tabular}{|l|l|l|l|l|l|l|l|l|l|l|l|l|}
\hline & T0 & Skill & T1 & Skill & T2 & Skill & T3 & Skill & T4 & Skill & T5 & Skill \\
\hline E0 & 0.5 & Java & 2 & VB & 2 & VB & 0 & Java & 1.5 & VB & 0.5 & Java \\
\hline E1 & 1.5 & Java & 1 & Java & 0 & null & 0.5 & Java & 0.5 & Java & 1.5 & Java \\
\hline E2 & 0 & null & 0 & null & 0 & null & 0 & null & 1 & C++ & 0 & null \\
\hline E3 & 0.5 & Java & 0 & VB & 0.5 & VB & 1 & Java & 0 & VB & 0.5 & Java \\
\hline E4 & 0 & C & 0 & null & 0 & null & 0 & C & 0 & null & 0 & C \\
\hline E5 & 1 & Java & 2 & VB & 1.5 & VB & 1 & Java & 0 & VB & 0 & Java \\
\hline E6 & 2 & Java & 2 & Java & 2 & null & 2 & Java & 2 & Java & 0.5 & Java \\
\hline E7 & 1.5 & Java & 1 & VB & 0.5 & VB & 2 & Java & 1 & VB & 1.5 & Java \\
\hline E8 & 2 & Java & 2 & Java & 2 & null & 2 & Java & 1 & Java & 1 & Java \\
\hline E9 & 0 & C & 0 & null & 0 & null & 0 & C & 1 & C++ & 1.5 & C \\
\hline Total & 9 & 0 & 10 & 0 & 8.5 & 0 & 8.5 & 0 & 8 & 0 & 7 & 0 \\
\hline
\end{tabular}

Table 8. An optimum Schedule 7: skill matching, all above objectives and constraints with overloading as soft constraint.

\begin{tabular}{|l|l|l|l|l|l|l|l|l|l|l|l|l|}
\hline & T6 & Skill & T7 & Skill & T8 & Skill & T9 & Skill & T10 & Skill & T11 & Skill \\
\hline E0 & 0 & VB & 0 & null & 2 & VB & 0 & Java & 0 & null & 1 & Java \\
\hline E1 & 0 & null & 1.5 & C & 1 & Java & 1 & Java & 2 & C & 1 & Java \\
\hline E2 & 1.5 & C++ & 0 & null & 0 & null & 0 & Null & 1 & C++ & 0 & null \\
\hline E3 & 2 & VB & 2 & VC++ & 0.5 & VB & 1 & VC++ & 0 & null & 1 & VC++ \\
\hline E4 & 0 & null & 1 & C & 0 & null & 0 & Null & 1 & C & 0 & null \\
\hline E5 & 0 & VB & 0 & null & 1 & VB & 2 & Java & 2 & C++ & 1 & Java \\
\hline E6 & 0.5 & C++ & 0 & null & 2 & Java & 2 & Java & 0 & C++ & 0.5 & Java \\
\hline E7 & 2 & VB & 0 & null & 1 & VB & 0.5 & Java & 2 & C++ & 2 & Java \\
\hline E8 & 0 & C++ & 0 & null & 2 & Java & 1 & Java & 1 & C++ & 0 & Java \\
\hline E9 & 0 & C++ & 1 & VC++ & 0 & null & 0.5 & VC++ & 1 & C++ & 0.5 & VC++ \\
\hline Total & 6 & 0 & 5.5 & 0 & 9.5 & 0 & 8 & 0 & 10 & 0 & 7 & 0 \\
\hline
\end{tabular}

5.2.8 Scope creeping plan / schedule type 8 /HRD plan . SCP plan requires the schedule of having minimum time and cost, and must follow working load. This type of schedule can be done when PM managers put restriction not only on quality but its feasible addition of features. Adding additional skilled employee, but not being given overload gives the scope creeping plan, but it gives another version of plan for the customers, also. Re-planning is the main purpose of this schedule as PM wants to add more and more skilled employee and gives various direction and scope of project. Finally, we gave same and equal weight age for the components of composite function.

This type of schedule considers minimum time and cost with overloading factor as hard constraint. Above all schedules may go beyond the overloading limit or capacity of particular employee. Above all schedule gives stress to the employee to complete within a total budget and total time without considering their speed and capability and stamina. But, this schedule type is another good example of optimization considering all the factors and assuming that every employee can do every task by limiting the overloading.

\section{RESULT AND DISCUSSIONS}

\subsection{About graphs and Tables}

Nearly all the schedule's fitness vs Generation graphs are shown in Fig.12, 13, 16, 17 where we can see after some generation (after 100 approximately) fitness gets it's steady positions. The fig.14 and fig.15 shows the graph of Time (Total Time) and Cost comparison with every schedules. But, critical time, GRGA CT time is given in the comparative table-11. Some schedules where the task completion is necessary gives graphs on Task Completion VS Generation.

- TT is taken in the graphs in figures indicates the total time to require to complete the all the tasks(in sequence). TT is the additions of all the task duration which represent the total effort requires for the whole project. The TT doesn't give the guarantee of completion task according to the definition of proposed approach for task completion.

- TC gives the critical time of the project for the particular schedule type. Even though TC is in the range of planned, it doesn't give the assurance of completing the task in defined time. But, according to Carl's definition of time completion that each task must have at least one employee. It results in increase in the percentage of task completion or nearly all tasks get completed. But, proposed approach implies tasks completion should have duration in defined rang.

In graphs (in figures) for the fitness Vs generation gives similar behavior of curve, moving from small values to high and make it steady after nearly 70 to 80 generations. The curve for the cost, total time, CT is approximately opposite to the fitness curve. After some generations (from the same point fitness make steady and straight) Cost, Time curve gets straight path but in downward side of $X$ axis with $\|$. In Multiple objective optimisation curves, the graphs for and after schedule type- 4 , the curves of objective components (TT, CT, TC, Cost) gives fluctuations in the curve; till it gets steady fitness value due to the Generepair utilization in proposed GRGA. It happens as it's characteristic is like an external mutation technique. We can see that if the hard constraint is task duration, then and only then, the TC curve gets 18 value, total 
Table 9. An optimum Schedule 7: skill matching, all above objectives and constraints with overloading as soft constraint.

\begin{tabular}{|l|l|l|l|l|l|l|l|l|l|l|l|l|}
\hline & T12 & Skill & T13 & Skill & T14 & Skill & T15 & Skill & T16 & Skill & T17 & Skill \\
\hline E0 & 2 & VB & 0 & null & 2 & VB & 0 & VB & 0 & null & 2 & Java \\
\hline E1 & 0 & null & 0 & null & 0 & Null & 0 & null & 0 & null & 0.5 & Java \\
\hline E2 & 0 & null & 0.5 & C++ & 0 & Null & 1 & C++ & 1 & C++ & 0 & C++ \\
\hline E3 & 2 & VC++ & 0.5 & VC++ & 1 & VC++ & 1 & VB & 1 & VC++ & 1.5 & Java \\
\hline E4 & 0 & null & 0 & null & 0 & Null & 0 & null & 0 & nul & 10 & null \\
\hline E5 & 1 & VB & 1 & C++ & 2 & VB & 0.5 & VB & 0.5 & C++ & 2 & Java \\
\hline E6 & 0 & null & 2 & C++ & 0 & Null & 0 & C++ & 0.5 & C++ & 0.5 & Java \\
\hline E7 & 2 & VB & 2 & C++ & 2 & VB & 2 & VB & 2 & C++ & 1.5 & Java \\
\hline E8 & 0 & null & 1 & C++ & 0 & Null & 1.5 & C++ & 1.5 & C++ & 0.5 & Java \\
\hline E9 & 1.5 & VC++ & 2 & VC++ & 1.5 & VC++ & 1 & C++ & 1 & VC++ & 0 & C++ \\
\hline & 8.5 & 0 & 9 & 0 & 8.5 & 0 & 7 & 0 & 7.5 & 0 & 8.5 & 0 \\
\hline
\end{tabular}

\begin{tabular}{|c|c|c|c|c|c|c|c|c|c|c|c|c|c|c|c|c|c|c|}
\hline \multirow{5}{*}{$\begin{array}{c}12 \\
10\end{array}$} & \\
\hline & \multicolumn{18}{|c|}{ Schedule type $7: C D \leq G R G A D \leq C P D$} \\
\hline & \multirow{2}{*}{\multicolumn{18}{|c|}{\begin{tabular}{|l|l|} 
\\
\end{tabular}}} \\
\hline & & & & & & & & & & & & & & & & & & \\
\hline \multirow{2}{*}{\multicolumn{19}{|c|}{ 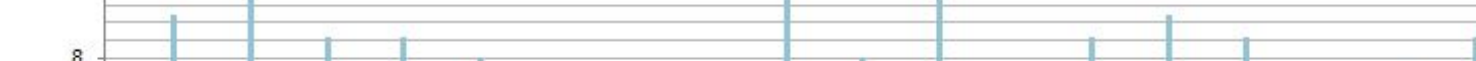 }} \\
\hline & & & & & & & & & & & & & & & & & & \\
\hline \multicolumn{19}{|l|}{8} \\
\hline & & & & & & & & & & & & & & & & & & \\
\hline \multirow{2}{*}{\multicolumn{19}{|c|}{6}} \\
\hline & & & & & & & & & & & & & & & & & & \\
\hline & & & & & & & & & & & & & & & & & & -5 \\
\hline \multirow{5}{*}{\multicolumn{19}{|c|}{4}} \\
\hline & & & & & & & & & & & & & & & & & & \\
\hline & & & & & & & & & & & & & & & & & & \\
\hline & & & & & & & & & & & & & & & & & & \\
\hline & & & & & & & & & & & & & & & & & & \\
\hline \multirow[t]{2}{*}{0} & II & & & & & & & & & & & & & & & & & 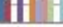 \\
\hline & 1 & 2 & 3 & 4 & 5 & 6 & 7 & 8 & 9 & 10 & 11 & 12 & 13 & 14 & 15 & 16 & 17 & 18 \\
\hline Empo & 0.5 & 2 & 2 & 0 & 1.5 & 0.5 & 0 & 0 & 2 & 0 & 0 & 1 & 2 & 0 & 2 & 0 & 0 & 2 \\
\hline -Emp1 & 1.5 & 1 & 0 & 0.5 & 0.5 & 1.5 & 0 & 1.5 & 1 & 1 & 1.5 & 1 & 0 & 0 & 0 & 0 & 0 & 0.5 \\
\hline Emp2 & 0 & 0 & 0 & 0 & 1 & 0 & 1.5 & 0 & 0 & 0 & 1 & 0 & 0 & 0.5 & 0 & 1 & 1 & 0 \\
\hline Emp3 & 0.5 & 0 & 0.5 & 1 & 0 & 0.5 & 2 & 2 & 0.5 & 1 & 0 & 1 & 2 & 0.5 & 1 & 1 & 1 & 1.5 \\
\hline -Emp4 & 0 & 0 & 0 & 0 & 0 & 0 & 0 & 1 & 0 & 0 & 1 & 0 & 0 & 0 & 0 & 0 & 0 & 0 \\
\hline Emp5 & 1 & 2 & 1.5 & 1 & 0 & 0 & 0 & 0 & 1 & 2 & 2 & 1 & 1 & 1 & 2 & 0.5 & 0.5 & 2 \\
\hline Emp6 & 2 & 2 & 2 & 2 & 2 & 0.5 & 0.5 & 0 & 2 & 2 & 0 & 0.5 & 0 & 2 & 0 & 0 & 0.5 & 0.5 \\
\hline$=\mathrm{Emp} 7$ & 1.5 & 1 & 0.5 & 2 & 1 & 1.5 & 2 & 0 & 1 & 0.5 & 2 & 2 & 2 & 2 & 2 & 2 & 2 & 1.5 \\
\hline Emp8 & 2 & 2 & 2 & 2 & 1 & 1 & 0 & 0 & 2 & 1 & 1 & 0 & 0 & 1 & 0 & 1.5 & 1.5 & 0.5 \\
\hline "Emp9 & 0 & 0 & 0 & 0 & 1 & 1.5 & 0 & 2 & 0 & 0.5 & 1 & 0.5 & 1.5 & 2 & 1.5 & 1 & 1 & 0 \\
\hline GRGAD & 9 & 10 & 8.5 & 8.5 & 8 & 7 & 6 & 6.5 & 9.5 & 8 & 9.5 & 7 & 8.5 & 9 & 8.5 & 7 & 7.5 & 8.5 \\
\hline
\end{tabular}

Fig. 9. Employee loading and task duration for Schedule Type 7

number of tasks in the project. Other schedule gives less than 18 as $\mathrm{TC}$ is soft constraint.

GAGR behaves nearly same manner in all types of schedule resulted as this can be seen in fig.11. The curved lines are nearly $\|$ to each others. Some tables in schedule's sections gives the dedication of employees to the tasks ( (All output tables of every schedule is with the authors)). It shows various GRSHD with respect to the schedule types. Some tables in the study section gives value of GR duration in the range defined where TC is must.

\subsection{Difference and similarities between our AND Carl, Mark and Tao's Approach [9]}

The Carl, Mark, Tao [9] defined genotype is 2D array assignment, one for employee and another for activity (task). New approach supports partial commitment. Proposed approach did the change in allele's values, so that, the gene which is not required may be removed from the evaluation itself before. This can be achieved by making the some of the chromosome as homozygote. Another solution is to keep value zero for all the alleles for particular locus of chromosomes. The skill which is not required for tasks for employee is kept zero. We kept the chromosome homozygotic for some alleles. Mathematically, we made hard constraint it self at the individual level. This is also called as external mutation 


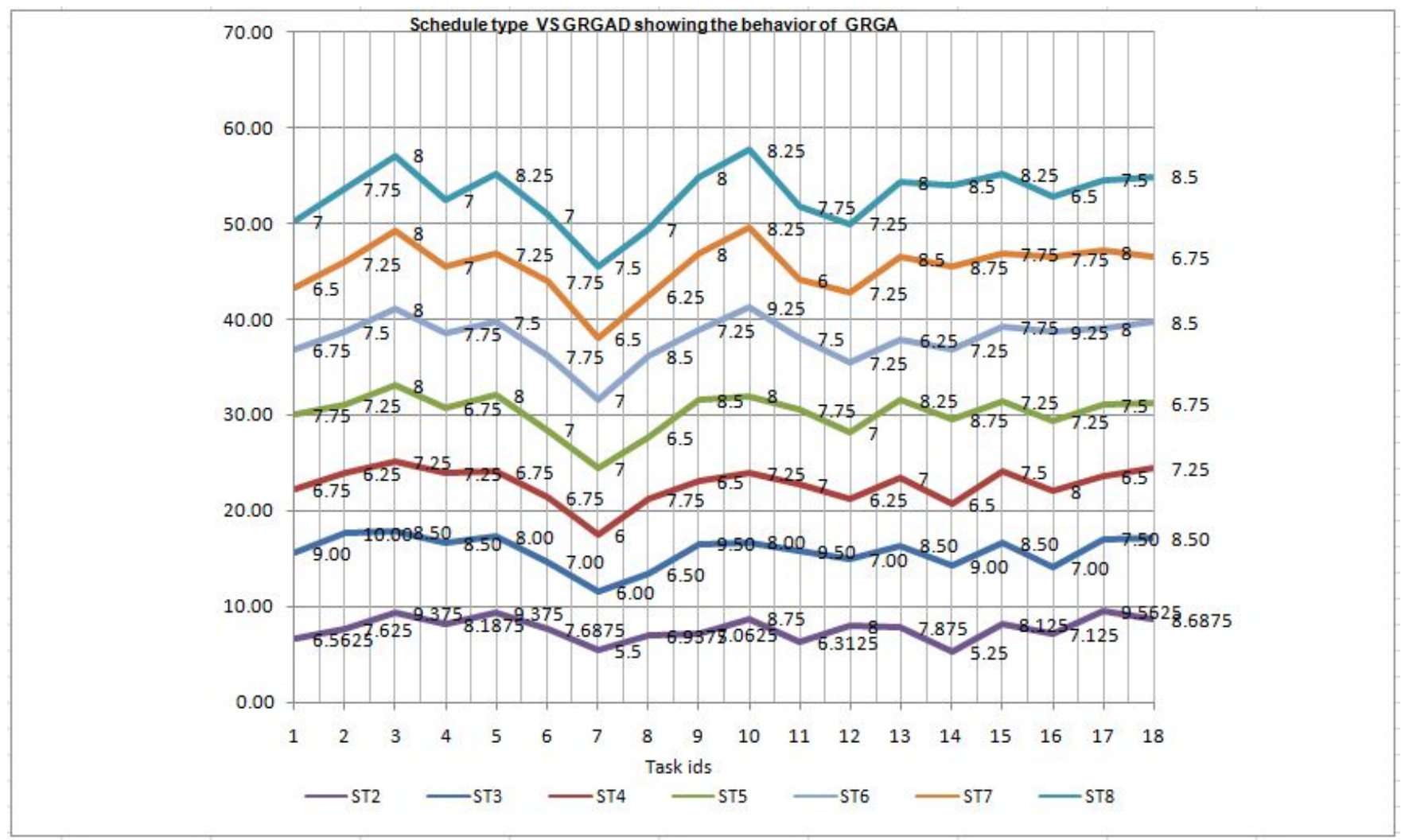

Fig. 11. Linear behaviour of GRGA

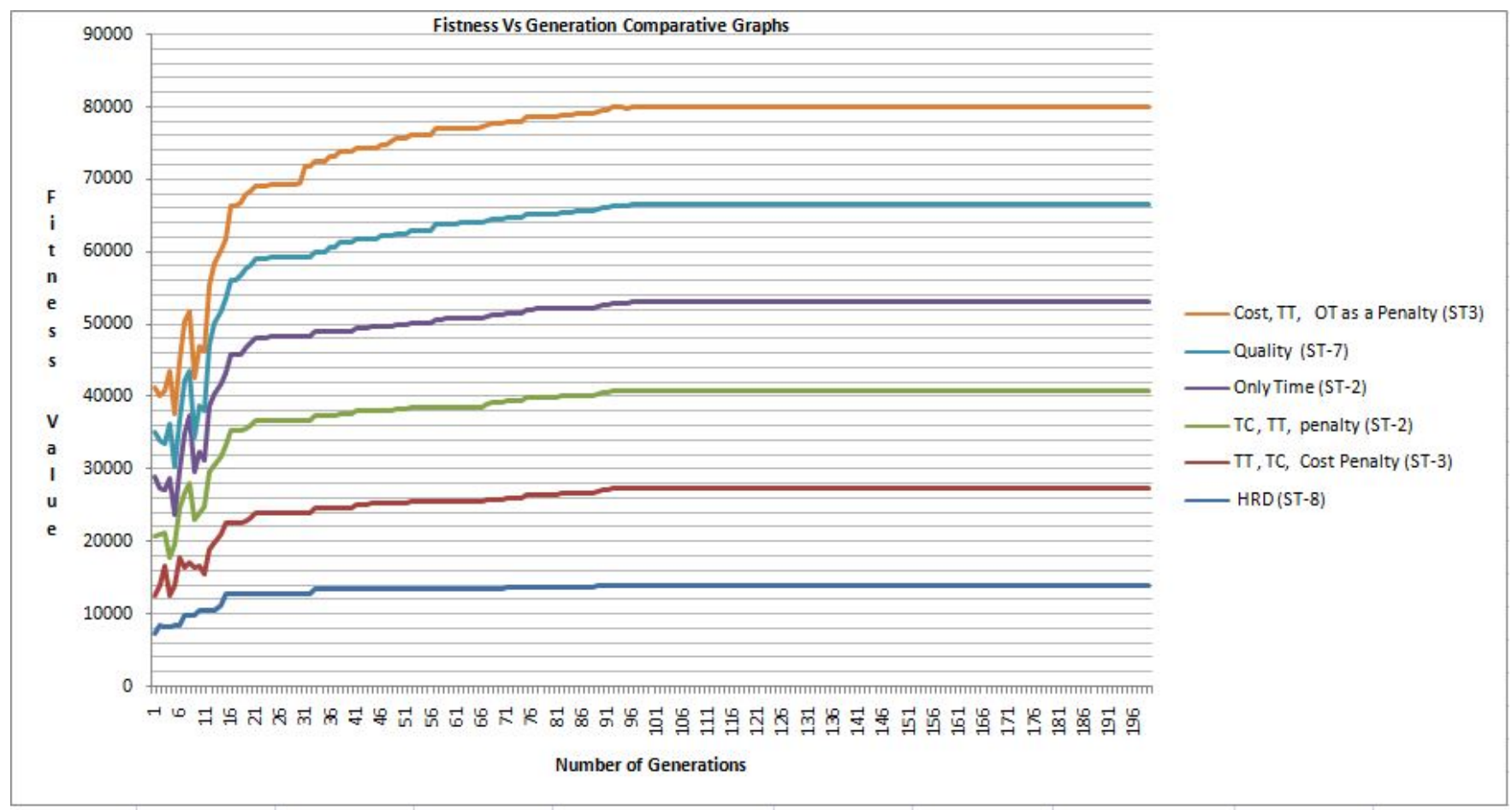

Fig. 12. Fitness Vs Generation 


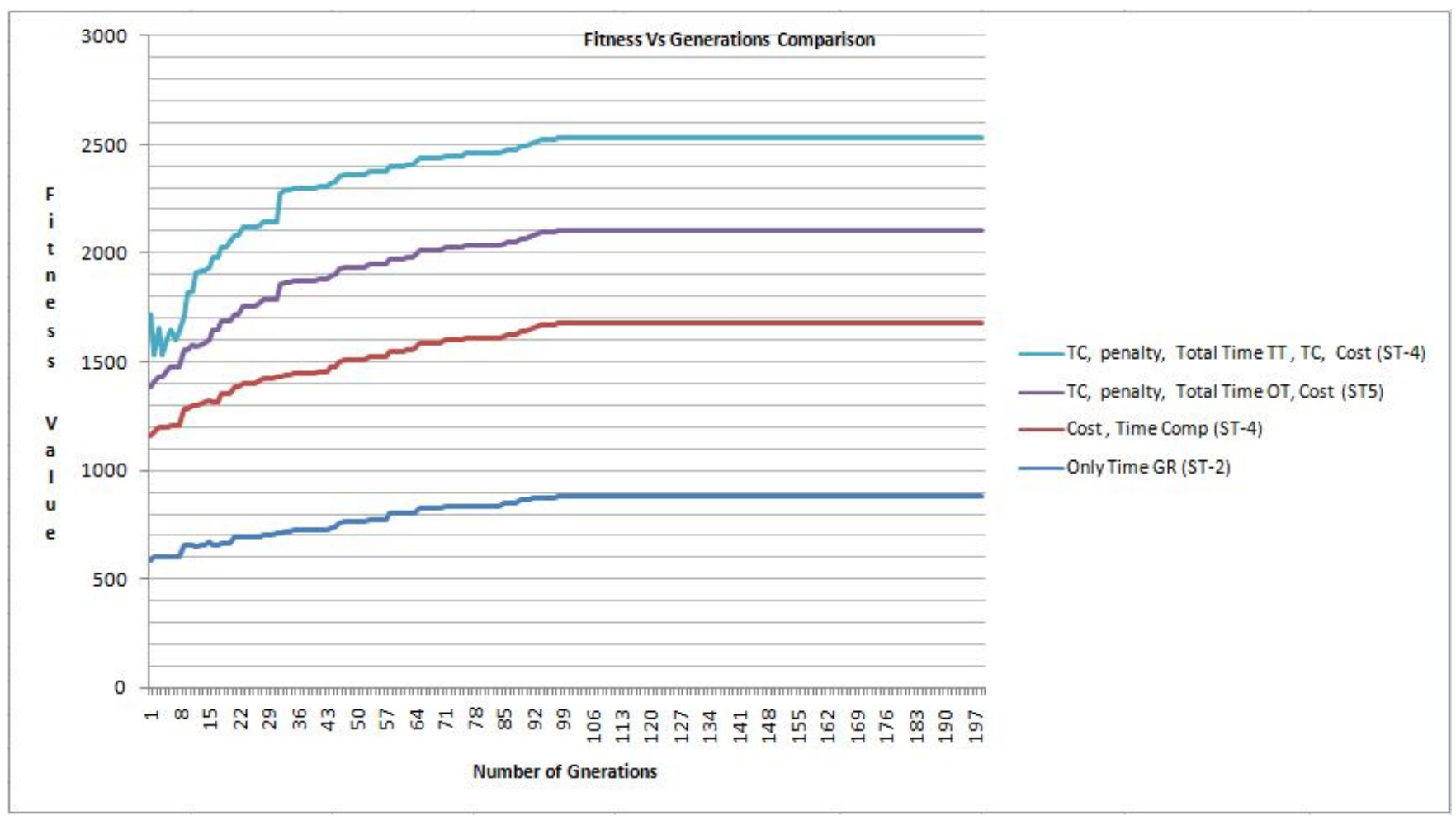

Fig. 13. Fitness Vs Generation

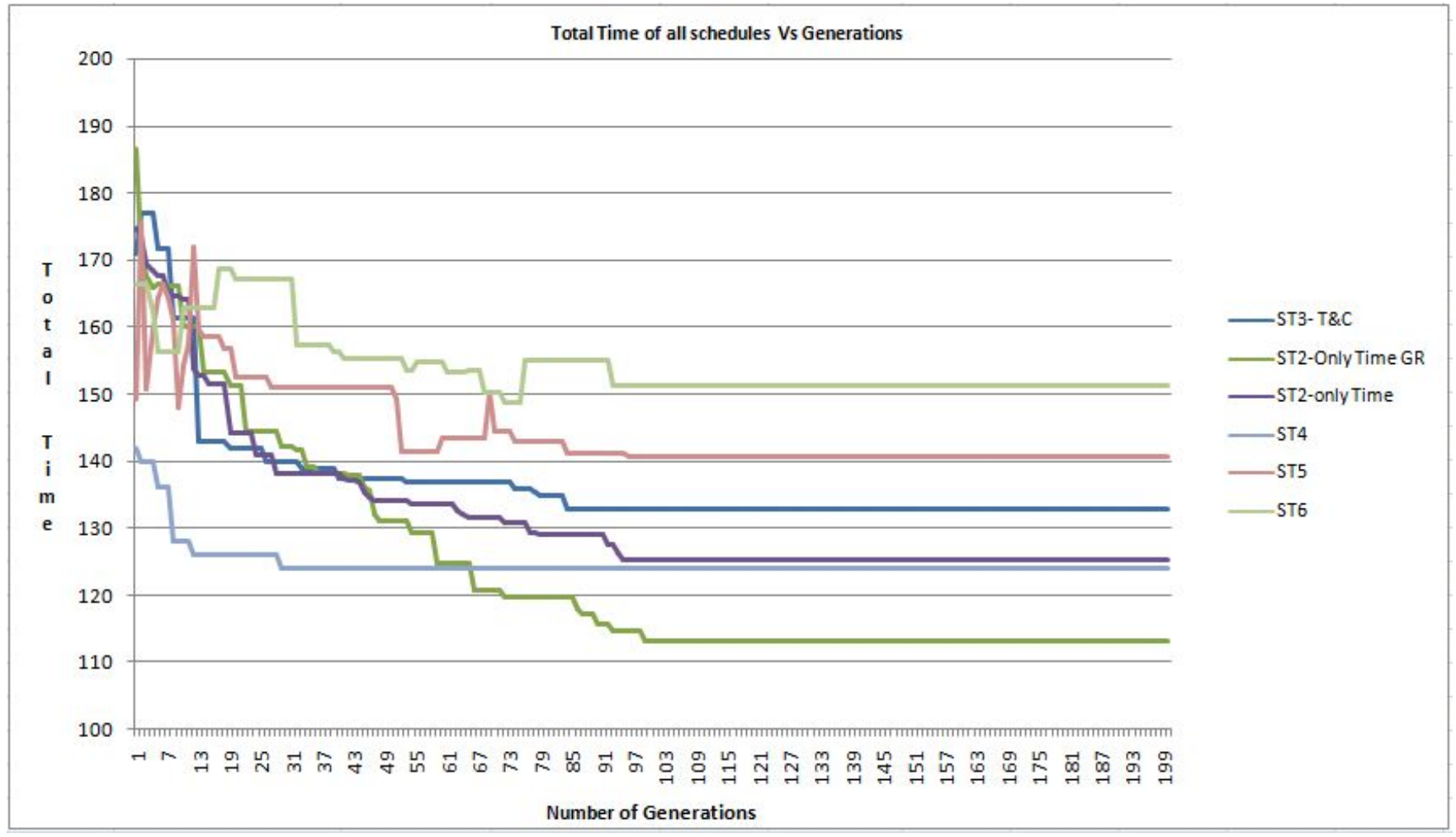

Fig. 14. Time comparison of all the schedules 


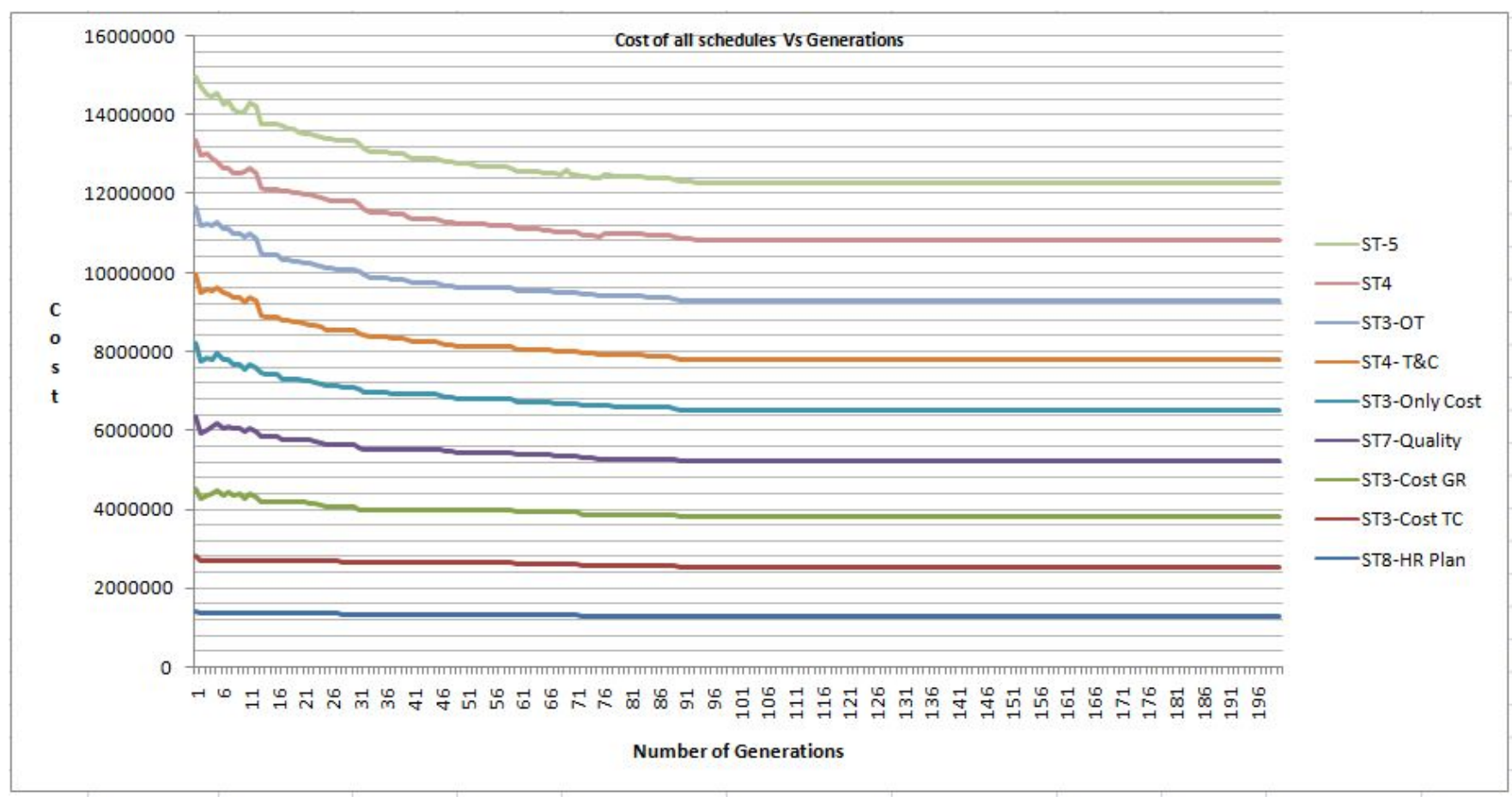

Fig. 15. Cost comparison of all the schedules

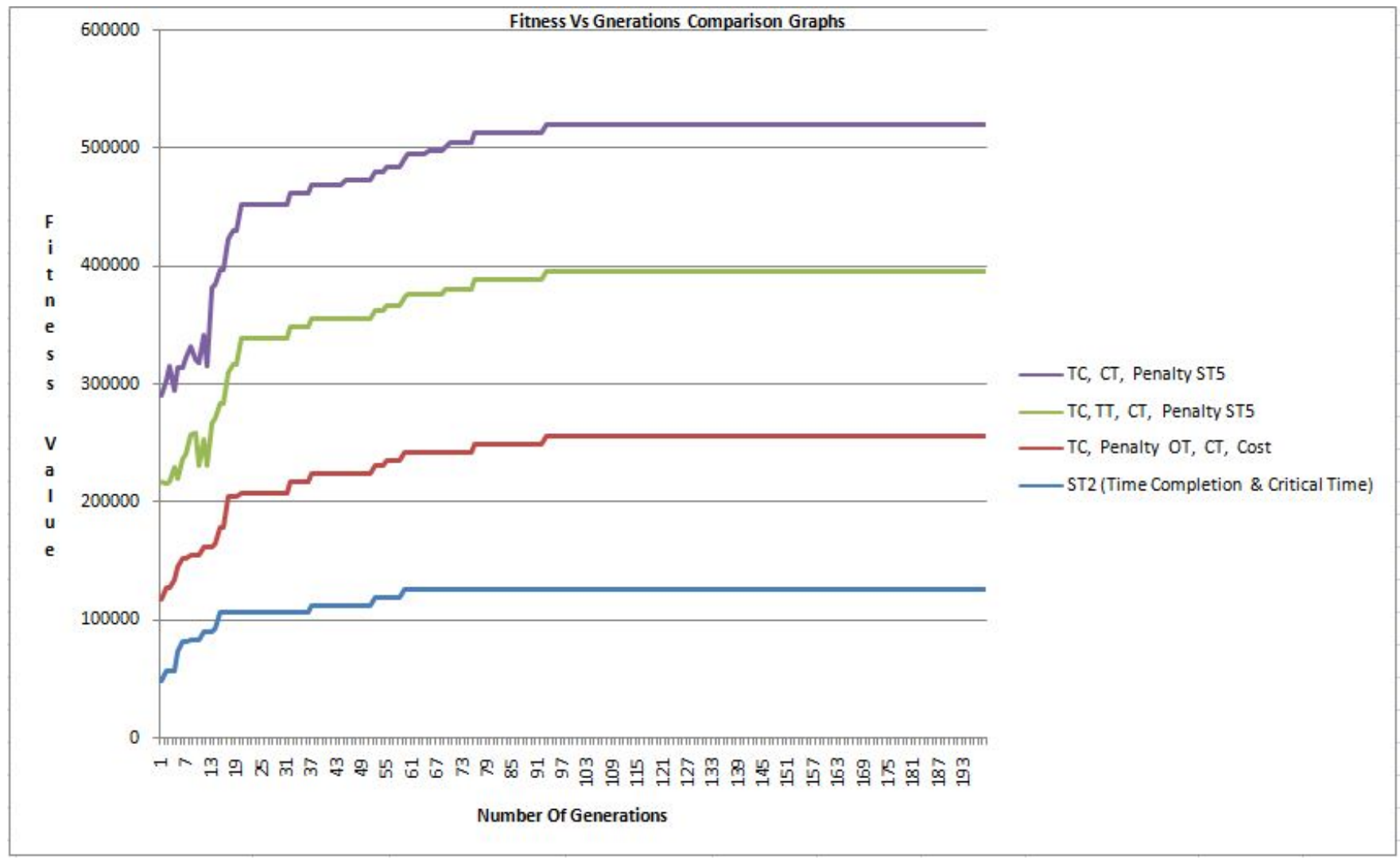

Fig. 16. Fitness Vs Generation 
Table 10. CD, CPD, PV, Rounded CHC (RCHC),Double of (RCHC+1)should be equal to GRGA apporach Head count as proposed approach uses concurrent tasks completion which are in $\|$.

\begin{tabular}{|c|c|c|c|c|c|c|c|c|c|}
\hline Task ID & Effort PM & $\mathrm{CD}$ & CPD & PV & $\mathrm{CHC}$ & $\mathrm{RCHC}$ & $\mathrm{RCHC}+1$ & Double(RCHC+1) & GRGA-HC-7 \\
\hline 0 & 10.00 & 6.00 & 9.60 & 51840 & 1.67 & 2.00 & 3.00 & 6.00 & 7.00 \\
\hline 1 & 15.00 & 7.00 & 11.20 & 60480 & 2.14 & 2.00 & 3.00 & 6.00 & 6.00 \\
\hline 2 & 20.00 & 8.00 & 12.80 & 69120 & 2.56 & 3.00 & 4.00 & 8.00 & 5.00 \\
\hline 3 & 10.00 & 6.00 & 9.60 & 51840 & 1.67 & 2.00 & 3.00 & 6.00 & 6.00 \\
\hline 4 & 15.00 & 7.00 & 11.20 & 60480 & 2.14 & 2.00 & 3.00 & 6.00 & 7.00 \\
\hline 5 & 15.00 & 7.00 & 11.20 & 60480 & 2.14 & 2.00 & 3.00 & 6.00 & 7.00 \\
\hline 6 & 10.00 & 6.00 & 9.60 & 51840 & 1.67 & 2.00 & 3.00 & 6.00 & 4.00 \\
\hline 8 & 20.00 & 8.00 & 12.80 & 69120 & 2.56 & 3.00 & 4.00 & 8.00 & 7.00 \\
\hline 9 & 20.00 & 8.00 & 12.80 & 69120 & 2.56 & 3.00 & 4.00 & 8.00 & 7.00 \\
\hline 10 & 10.00 & 6.00 & 9.60 & 51840 & 1.67 & 2.00 & 3.00 & 6.00 & 7.00 \\
\hline 11 & 15.00 & 7.00 & 11.20 & 60480 & 2.14 & 2.00 & 3.00 & 6.00 & 7.00 \\
\hline 12 & 20.00 & 8.00 & 12.80 & 69120 & 2.56 & 3.00 & 4.00 & 8.00 & 7.00 \\
\hline 13 & 25.00 & 8.50 & 13.60 & 73440 & 2.94 & 3.00 & 4.00 & 8.00 & 7.00 \\
\hline 14 & 15.00 & 7.00 & 11.20 & 60480 & 2.14 & 2.00 & 3.00 & 6.00 & 5.00 \\
\hline 16 & 15.00 & 7.00 & 11.20 & 60480 & 2.14 & 2.00 & 3.00 & 6.00 & 5.00 \\
\hline 17 & 10.00 & 6.00 & 9.60 & 51840 & 1.67 & 2.00 & 3.00 & 6.00 & 6.00 \\
\hline Total & 265.00 & 124.50 & 199.20 & 1075680 & 12.72 & 13.00 & 14.00 & 28.00 & \\
\hline
\end{tabular}

Table 11. Comparison between GRGA \& Carl's duration.

\begin{tabular}{|c|c|c|c|c|c|c|}
\hline Schedule Type & GRCT (PV) & Salary & Schedule Cost & EV & CPI/SPI & Carl Result [9] \\
\hline ST3 (Cost \& Task completion) & 31 & 54000 & 1400012.5 & 32 & 1.032258065 & $*$ \\
\hline ST3 (only Cost but GR ) & 30 & 54000 & 1289013.5 & 32 & 1.066666667 & $*$ \\
\hline ST2 (only Task Completion) & 31 & 54000 & $*$ & 32 & 1.032258065 & $*$ \\
\hline ST2 (Only Time, GR) & 14.5 & 54000 & $*$ & 17 & 1.172413793 & $*$ \\
\hline ST3 Simple cost & & 54000 & 1402011 & 1550000 & 1.105554807 & $*$ \\
\hline ST2 Simple Time & 13.75 & 54000 & $*$ & 17 & 1.236363636 & 14 \\
\hline ST2 TC,CT and Time Penalty & 29 & 54000 & $*$ & 32 & 1.103448276 & $*$ \\
\hline $\begin{array}{l}\text { ST3 (Cost,Task Completion } \\
\text {, OT as a Penalty) }\end{array}$ & 30 & 54000 & 1462011 & 32 & 1.066666667 & $*$ \\
\hline $\begin{array}{l}\text { ST2 (Task Completion \& } \\
\text { Critical Time) }\end{array}$ & 30 & 54000 & $*$ & 32 & 1.066666667 & $*$ \\
\hline $\begin{array}{l}\text { ST3 Cost Overtime and } \\
\text { Task completion }\end{array}$ & & 54000 & 1443011 & 1550000 & 1.074142886 & $*$ \\
\hline ST4 ( Penalty OT CT Cost) & 28 & 54000 & 1534007 & 32 & 1.142857143 & $*$ \\
\hline ST5 & 30 & 54000 & 1478008 & 32 & 1.066666667 & $*$ \\
\hline $\begin{array}{l}\text { ST6 Unit time and } \\
\text { Price schedule }\end{array}$ & 30 & 54000 & 847800 & 32 & 1.066666667 & $*$ \\
\hline ST7 Quality plan & 31 & 54000 & 1416007 & 32 & 1.032258065 & 35 \\
\hline ST8 HR Plan & 25.625 & 54000 & 1265512 & 32 & 1.248780488 & 28 \\
\hline
\end{tabular}

\section{technique.}

Carl, Mark and Tao [9] used the rejecting strategy, discards all infeasible chromosomes created throughout an evolutionary process. Although, this strategy is well popular in many GA. The method gives satisfied result when search space is convex and it constitutes a reasonable part of the whole search space. However, such an approach has serious limitations. For example, for many constrained optimization problems where the initial population consists of infeasible chromosomes only. It might be essential to improve them. Moreover, quite often the system can reach the optimum easier if it is possible to cross an infeasible region (especially in non-convex feasible search spaces). We used penalty, reward method keeping in mind the invalid solution may have good alleles for schedules (type4 and onwards). These strategy has the advantage that they never generate infeasible solutions as for highly constrained problem, infeasible solution may take a relatively big portion in population.

Repairing an infeasible chromosome returns feasible solution and generate a feasible one through repairing procedure. For many combinatorial optimization problems, it is relatively easy to create a repairing procedure. Repairing strategy depends on the existence of a deterministic repair procedure to converting an infeasible offspring into a feasible one. In our approach, we made change in the genes according to the summation of the column so that we get GRGAD in between pessimistic and optimistic. Pushing the other alleles, gene values, into another locus (where it should have) made the schedule tend to parallel for reducing the time required for project. Though, we obtained some heterozygoses but, had 


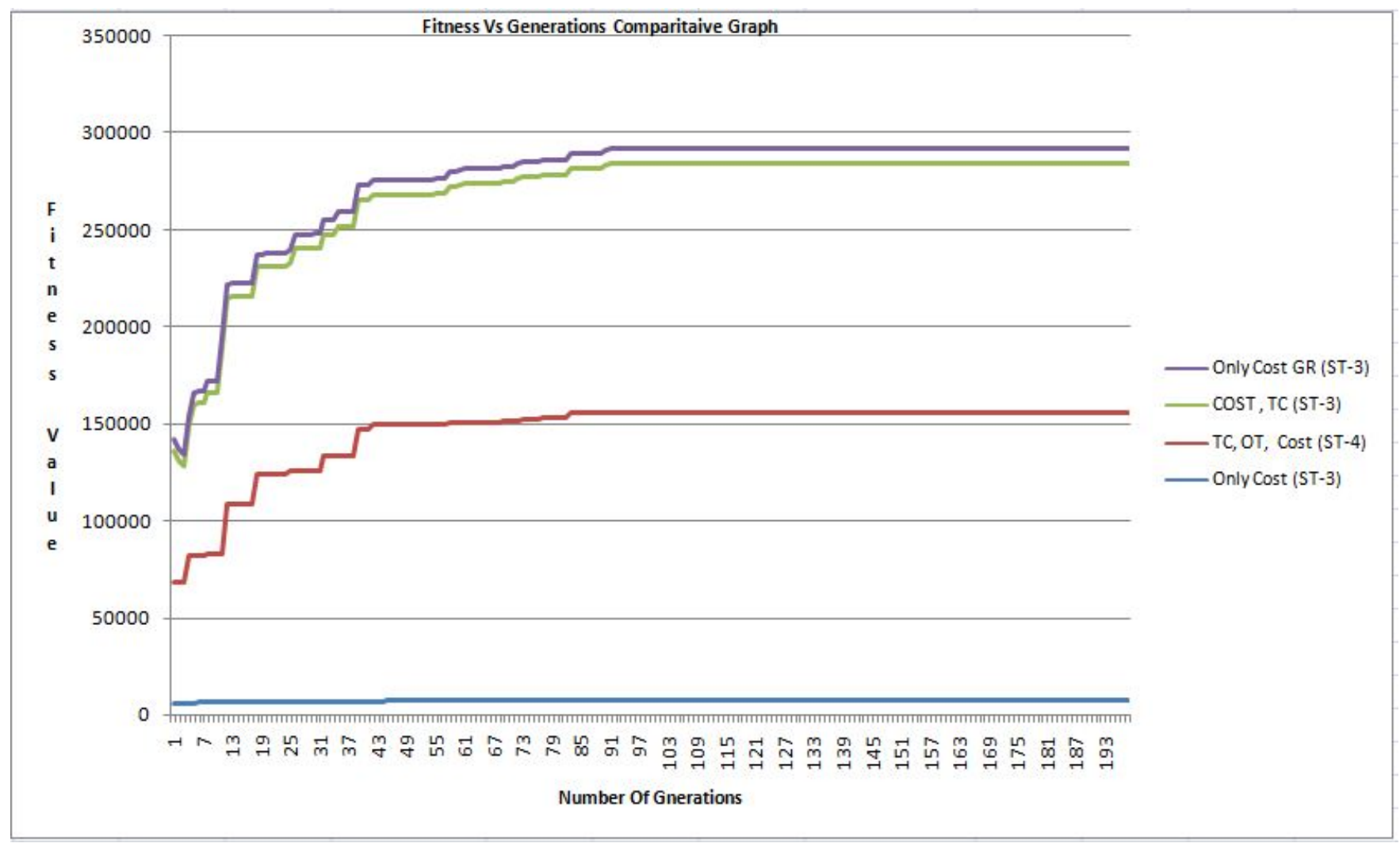

Fig. 17. Fitness Vs Generation

same fitness.

Objective functions are not isolated from genetic operations as Carl, Mark, Tao made two division of the objective functions one is major and another is minor part. Employees are assigned partial, discrete allocation of time from $\{0,0.25,0.5,0.75,1\}$. The major part Carl includes task completeness and skill matching, are made must. We have taken this as not only hard constraint but also we changed the definition of "task completion". One should say Task is completed iff

$$
C D_{T_{i}} \leq G R O D_{T_{i}} \leq C P D_{T_{i}}
$$

We compared our value duration with Carl's results of duration and else [9]. In proposed seventh schedule type, minimizing the time and matching the skill is exactly opposite objectives, so the objectives are in opposite directions. We have solved the problems for schedule type 1 to schedule type 8 and reformulated two and more than two objectives.

We created skill mapping of task and employee matrix which tells skill of each with employee. The matrix mapping of $\mathrm{TS}_{i j}$ and $\mathrm{ES}_{k j}$ is done for getting another matrix $\mathrm{TES}_{T_{i} S_{i}}$ for getting the solution in less time and generation. Evolving process as the objective function is isolated from genetic operating process in Carl's approach. The objective function is integrated into the genetic operators in proposed approach. Only objective function needs to be modified for different projects. Both operators and objective functions are not needed to be modified for different projects in our and Carl's approach. GRGA approach normalises the objective values time and cost by dividing it by it's max value in that generation.

Carl GA used normalized objective values ;Because, different objectives can have different scales, it would be more meaningful to normalize their values before evaluation and comparison. All objective values (cost, time) were normalized into the range of $\{0,1\}$. Weighting was not effective for two objective values on two totally different scales. A composite objective can be used, by summing weighted objective values. Gene repairing, homozygosis of some offsprings gives the solution very fast and optimal in GRGA approach. JAVA Classes gives various function which are used with our defined class code. The proposed approach uses $\mathrm{SM}$ and $\mathrm{CM}$ for the stoping criteria for every schedules except schedule type-1.

The solutions from genetic algorithms solved by our approach, after some generation, starts to give the feasible solutions in terms of minimization of objective function defined. The proposed approach is the combination of various types of constraints module to get the optimized solution by incorporating Langrages multipliers normalization of variables and giving weight age to the different variables. Nearly all the schedules except ST-7 and ST-8, all the employees are having all the skills required as for the five process groups. If some one takes into account each employee having all skilled owned, the TPG becomes like chain. As any employee can do any task, any employee can be adjusted for any task in the project. The concurrency execution of parallel tasks result in 
less time. Nearly, $(3 / 4)^{t h}$ time of the project will be required to complete the other type of project like ST-7 and ST-8. So, the ST-2 with minimum time objective gives approximately half time of other schedule. The comparison table in terms of time, cost, total time, Task completion is shown in the table-11. $\mathrm{HC}_{T_{i}}$ i.e. team size for every schedule according to the COCOMO-II and GRGA is given. The team size more than double requires where the all employee possesses all skills due to parallel implementation of tasks. Due to the task completion is dependant on the team size as per our proposed definition, the team size required to complete in ST-7 and ST-8 is more than double, but less than the Carl results team size. GRGA uses the skilled parallel employees in the schedules. Other schedules give some what minimum results due to the parallelization but imparts less than critical time of the project.

Another way of calculating the CT (with TPG) is shown in the Fig.20 and Fig.21. It shows if we use slots for the allocation of employees to every task, we get the less time than the value of GRCT. This shows 83 slots i.e. $83 / 4$ as we use gene values $1,2,3,4,5$ for $0,0.25,0.5,0.75,1.0$ as dedication and devotion to the task from the employee side. It comes out to be approximately 21 months. This is due to the concurrency utilisation of employee in the the schedule.

\subsection{Combinational flavour of Classical and Genetic Algorithm for Optimization}

Classical optimization techniques are used for single variable constraints, multiple variable functions with equality constraints. Various problems of equality constraints are solved by Langrages multiplier method and inequality constraints problem by Kuhn-Tucker conditions. We made the combinations of linear programming, integer programming, stochastic programming, combinatorial optimization and constraint satisfaction with genetic algorithm. In proposed approach (linear programming) objective function ' $\mathrm{f}$ ' is linear and the set $\mathrm{E}, \mathrm{T}$, and $\mathrm{S}$ are the linear equalities. The genetic algorithm is itself a stochastic programming so naturally some of the constraints are dependent on randomization of crossover, mutation, selection. Another important aspect, we used is satisfying the hard constraints at the level of representation of a chromosome itself. This idea gives to make little search space with required hard constrained chromosome as an element of hypothesis Ultimately, we can start with the powerful pool of chromosomes to get the near optimal solutions within a less generation and less time.

The task completion is related to PM. Unless and until task duration doesn't go to the optimistic value duration, we can say that, not only task is incomplete, but also, the task is not done in quality manner. We strictly stick to the condition above mentioned for nearly all schedules produced. Not only, we achieved target schedule in less time but also in different objective conditions and in less generation by keeping some chromosome with duplicate individuals and some genes by repaired technique. This gives fast convergent to get optimal solution in the less time. This can be seen in table of comparison in Table-11 and Fig.19.

Keeping all employee busy is the natural approach of the management and it has to be. As we are considering FSPs as an employee, but, Carl used the some relaxation in the assignment of task to an employee. Carl ,itself, says that there is no relation between objective components and GA operations, but we didn't keep like this. So,every component is dependent on other gives

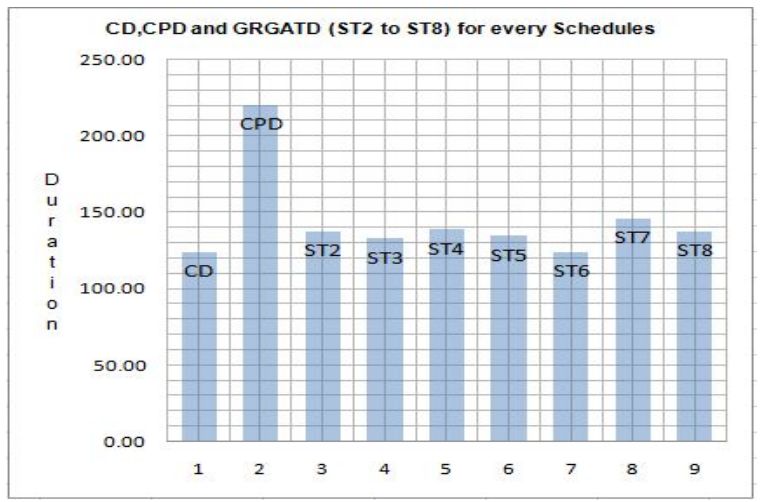

Fig. 18. GRGA total duration for schedule types

the more realistic optimal solution i.e heterozygote. We achieved less schedule cost and time than Carl's result as we made changes in the structure of chromosome itself before entering into the GRGA operations in the pool. Carl had explained it's objective components with weighted multiplier but we gave equal weight to every components of the objective function.

This article tries to adopt to complement the PMIs standard of management for scheduling of software development projects and programs. The PMI standard is really as being a project management reference that can be applied most of the time to most of the projects to get the success chances could be greatly increased. The five Project Management Process Groups highlight the integrative nature of projects and project management. Contract type selection is the most volitive and crucial establishing step in a business agreement with another party. This step may give possible and indirect hidden engagement risks. Therefore, companies should be very careful to get into contracts so that there is a minimum risk for their future business. It is always assumed and a good idea to have engagement in fixed bids whenever short-termed and predictable project is in business.

\subsection{Contract, discussion, directions and related work}

Prior research shown that, on average, vendors have higher profits associated with time and $\operatorname{Cost}(\mathrm{T} \& \mathrm{C})$ or $\mathrm{T} \& \mathrm{M}$ contracts than fixed price (FP) contracts. Gopal and Konduru research gives hints to the the relative importance of various profit drivers different across all contracts. Anandasivam Gopal and Konduru (2008) addressed this hint by using data on 93 offshore projects completed by a leading Indian vendor [5]. He gave hints to do the direction in terms of an endogenous switching regression framework and the program evaluation methodology to show that profit equations are distinctly different for the different contractual domains. They used the following two profit equations,

$$
\operatorname{Pr}(T M)=f\left(C, T O, D U R, \text { Team, EF, Prior }, \lambda_{T_{i}}\right)+V_{T_{i}}
$$

$$
\operatorname{Pr}(F P)=f\left(C, T O, D U R, T e a m, E F, \text { Prior }, \lambda_{F_{i}}\right)+V_{F_{i}}
$$

We can change to the following equations to get the profit of contracts. 
$\operatorname{Pr}(T D C)=f\left(C, T O, G R T C, G R H C, E F\right.$, Prior,$\left.\lambda_{T_{i}}\right)+V_{T_{i}}$

$$
\operatorname{Pr}(C D C)=f\left(C, T O, G R T C, G R H C, E F, \text { Prior }, \lambda_{F_{i}}\right)+V_{F_{i}}
$$

$\mathrm{Pr}=$ profit, $\mathrm{C}=$ Constant $\mathrm{TO}=$ Turnover, Prior $=$ History of clients, $\mathrm{CDC}=$ Cost oriented contract, $\mathrm{TDC}=$ Time oriented Contract. where The $\lambda$ terms are the inverse Mills ratios, and are calculated using parameters from the profit model estimated [5]. Gangadharrao Soundalyarao "G. S." Maddala [36, Indian American economist and mathematician, derived $\mathrm{V}_{T_{i}}, \mathrm{~V}_{F_{i}}$. These are random error terms with zero expectations. We may go for future direction to identify contingencies under which the vendor prefers which contract. This may be done by taking the all the factors as trait in the chromosome. The trait may be duration of project, Team size, employee turnover, prior and efforts. We have already considered the 3 properties for the contracts. But, others two are also important to ge the better results in software project scheduling management using EVM. As these factors are very important, since previous study says something about this factor as follows.

* DUR (Project duration) is a significant risk and volitant factor; longer the projects results in more risky turnout(Pressman 1992).

$* \mathrm{TPHC}_{s}$ is the size of the core team of the project. The core team plays instrumental role in determining both project costs and team productivity (Banker et al. 1991).

* High attrition rates, employee turnover, in Indian software development firms are now common (Nidumolu and Goodman 1993). It is never easy to measure the effects of attrition accurately because employee occupy different standards and levels of importance in a on going team development structure.

* Ethiraj et al. (2005) argue that prior experience with the same client make suitable environment and enables the vendor to get valuable insight. Hence, The learning into the clients business processes makes easy. Thus the ability and efficiency of vendor are enhanced. Therefore, this lead to more and more profitability in terms of cost, time and relations for the projects.

\section{CONCLUSION WITH FURTHER ISSUES}

Our proposed approach gives emphasize on the gene repair technique. Gene repair technique is used at the time of selection. The more effective and time efficient way is the change in the structure of chromosome representation where we put directly zero for skill matching in the structure itself so that search space is reduced to the skill matched space only for schedule type-7. Every employer, cost estimator want the employee has to work for some time as employer can not bear the cost of FST in small scale industries.

That problem can be solved by putting hard constrained as every employee must work for at least 2 weeks or 1 months for the particular tasks to maintain the quality in the work. That is achieved by keeping less number of values of genes or by less granularity of the chromosome value. GA with linear fitness function used single variable and metavariable gives better result with proper penalty and reward function. Even though, we got same fitness chromosomes, there are various genotypes individuals available for selection to PM. Our future work will be based on the group management and team management so that we can get more and more optimised solution with respect to time and cost by using various models. These models are related with team management. Benjamin Franklin says, 'All mankind is divided into three groups: those that are immovable, those that are movable, those that move'. This is equally applicable for SPM ( software project management) as the time line based schedule depends upon the skills of FSP, their experience,their speed of work and psychological conditions. All these factors are of these three category people with which we have to cope up and achieve milestones of entire project in systematic way.

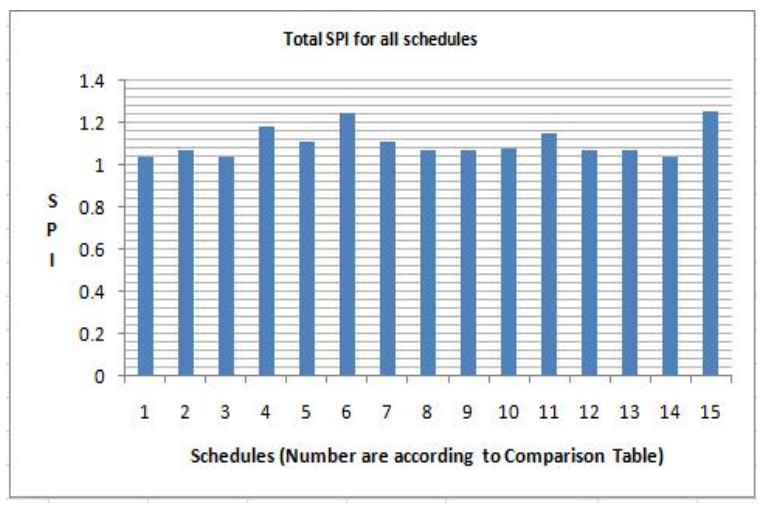

Fig. 19. Total Margin allowed for every schedule types i.e. margin is above 1.0 value of SPI

We have to make the team and group of more and more movable people rather than immovable by making them trained in SPLC itself. The schedules considered and produced are indirectly connected with different features (Quality factors and COCOMO-II cost drivers). These features can be the objective function variables. These features and types of contracts, these features (Quality factors and COCOMO-II cost drivers) and indirect costs, these features and phase wise plan, these features and other types of plan can output the different combination of schedule plan. All Quality drivers can be considered to calculate the BAC. Because, these drivers are related with CPI, SPI, CV, $\mathrm{SV}$ also. The Phase wise Expenditure or weight for Conventional SE in \% given by Walker can be used for prediction of $\mathrm{PV}, \mathrm{SV}$ and in return we will get the SPI,CPI in advance for target schedule for every phase of SPLC so that we can remove pregnant process phase wise and we can reduce the rework in SPM.

\section{CONCERNED READING, WORK AND INSPIRATION}

The research article written by Santosh Shinde [48] [46] gives the touch to the project management aspect. Software Team Formation for software project management by Neural Networks \& DTL is put as simple abbreviated prototype in this article [48]. The idea of Project Management by DTL is kept in front of us by the Santosh and his time in [46]. The book written by Amit and Dinesh [12] give help in the analysis of algorithm and it's design in the published papers [18] [17]. Other side, the book written by Mangesh and team $[26]$ is used to collect the related information regarding object oriented software engineering and design for the future 


\begin{tabular}{|c|c|c|c|c|c|c|c|c|c|c|c|c|c|c|c|c|c|c|c|c|c|c|c|c|c|c|c|c|c|c|c|c|c|c|c|c|c|c|c|c|c|c|c|c|c|}
\hline \multicolumn{46}{|c|}{ s in ST- 5 type sche } \\
\hline & \multicolumn{3}{|c|}{ Month1 } & \multicolumn{4}{|c|}{ Month2 } & \multicolumn{3}{|c|}{ Month3 } & \multicolumn{4}{|c|}{ Month4 } & \multicolumn{4}{|c|}{ Month5 } & \multicolumn{4}{|c|}{ Month 6} & \multicolumn{4}{|c|}{ Month7 } & \multicolumn{3}{|c|}{ Month8 } & \multirow{2}{*}{\multicolumn{4}{|c|}{\begin{tabular}{|l|l|l|l|l|l|l|l|l|l|}
\multicolumn{3}{|l|}{ Month } &
\end{tabular}}} & \multirow{2}{*}{\multicolumn{4}{|c|}{ Month10 }} & \multicolumn{4}{|c|}{ Month11 } & \multicolumn{4}{|c|}{ Month12 } \\
\hline & 1 & & 3 & \begin{tabular}{l|l}
4 & 5 \\
\end{tabular} & 6 & & 8 & \begin{tabular}{|l|l|}
9 & 10
\end{tabular} & 11 & 12 & 13 & & 15 & & 17 & 18 & 19 & 20 & 21 & & 23 & 24 & 25 & & & & 29 & & & & & & & & & & 40 & & & 43 & 44 & & & \begin{tabular}{l|l}
47 & 4.
\end{tabular} & \\
\hline & & & 6 & \begin{tabular}{l|l}
6 & 7 \\
\end{tabular} & 1 & 7 & 7 & \begin{tabular}{ll|}
7 & 1 \\
\end{tabular} & 1 & 1 & 1 & 1 & 1 & 1 & 1 & 1 & 1 & 1 & 1 & 1 & 1 & 1 & $1]$ T & & 1 & 1 & 1 & 1 & 1 & 1 & 1 & 1 & 1 & 1 & 1 & 1 & 1 & 1 & + & & 1 & & 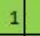 & & \\
\hline & 7 & & 7 & \begin{tabular}{l|l}
7 & 6
\end{tabular} & 6 & 6 & 6 & $\begin{array}{ll}6 & 7 \\
\end{array}$ & 6 & & $\mathrm{TT}$ & & & & 6 & & 6 & 6 & 6 & 6 & 6 & 6 & 6 & 6 & 6 & 6 & $6 \mathrm{~T}$ & & 6 & 6 & 6 & 6 & 7 & T & 7 & & & 7 & & $\mathrm{~T} 11$ & 6 & 6 & 6 & & \\
\hline & 8 & 8 & 8 & 88 & 8 & 8 & 8 & $\begin{array}{ll}8 & 8 \\
\end{array}$ & 8 & 8 & 8 & 8 & 8 & 8 & 8 & 8 & 8 & 8 & 8 & 8 & 8 & & & & 8 & 8 & 8 & 8 & 8 & 8 & 8 & 8 & 8 & & 8 & 8 & 8 & 8 & 8 & 8 & 8 & 8 & 8 & 8 & \\
\hline & 9 & 9 & 9 & 99 & 9 & 9 & 9 & \begin{tabular}{l|l}
9 & 9 \\
\end{tabular} & 9 & 9 & 9 & 9 & 9 & 9 & 9 & 9 & 9 & 9 & 9 & 9 & 9 & 9 & 9 & 9 & 9 & 9 & 9 & & & & & & 9 & & 9 & 9 & 9 & 9 & 9 & & 9 & 9 & 9 & 9 & \\
\hline & \begin{tabular}{|l|l|l|}
10 & 1 \\
\end{tabular} & 101 & 101 & 10 & 10 & & & & $\mathrm{T3}$ & & & & & & 10 & 10 & 10 & 10[ & $T 4$ & & & & & & & & & & & & & & & & & 6 & 6 & 6 & 6 & 6 & & & & & \\
\hline & 1 & 1 & 1 & 11 & 1 & 1 & 1 & 1 T5 & 3 & 3 & 0 & 3 & 3 & 3 & 3 & 3 & 3 & 3 & 3 & 3 & 3 & & & & 3 & 3 & 3 & 3 & & & & & 3 & 3 & & 3 & 3 & 3 & 3 & 3 & 3 & & 9 & & \\
\hline & 2 & $2^{2}$ & 2 & 2 & 2 & 2 & 2 & $\begin{array}{ll}2 & 2 \\
\end{array}$ & & & & 2 & 0 & 0 & 2 & 2 & 2 & 2 & 2 & 2 & 2 & 2 & & & 2 & 2 & 2 & 2 & & & 2 & 2 & 2 & 2 & $\begin{array}{ll}2 & 2 \\
\end{array}$ & 2 & 2 & 2 & & $\mathrm{T15}$ & 2 & 2 & 2 & 2 & 2 \\
\hline & 3 & 3 & 3 & 33 & 3 & 3] & 3 & \begin{tabular}{l|l}
3 & 3 \\
\end{tabular} & & & & 10 & 10 & 10 T & 10 & & & & & & & & & & & & & & & & & & & & & 5 & 0 & 0 & 7 & & , & & & & 0 \\
\hline & 4 & 4 & 4 & \begin{tabular}{l|l}
4 & 4 \\
\end{tabular} & 4 & 4 & 4 & \begin{tabular}{l|l}
4 & 4
\end{tabular} & 4 & 4 & 4 & 4. & 4 & 4 & 4 & 4 & 4 & 4 & 4. & 4. & 4 & 4 & 4 & 4 & 4 & 4 & 4 & 4 & 4 & 4 & 4 & & 4 & 4 & 4 & 4 & 4 & 4 & 4 & 4 & 4 & 4 & 4 & 4 & 4 \\
\hline & 5 & 5 & 5 & 55 & 5 & 5 & 5 & \begin{tabular}{l|l}
5 & 5 \\
\end{tabular} & 5 & 5 & 5 & 5 & 5 & 5 & 5 & 5 & 5 & 5 & 5 & 5 & 5 & 5 & 5 & 5 & 5 & 5 & 5 & 5 & 5 & 5 & 5 & 5 & & & & & 5 & 5 & 5 & & 5 & 5 & & 5 & 5 \\
\hline$\frac{b}{H}[$ & & & & & & $\mathrm{T1}$ & & \begin{tabular}{l|l}
0 & 0 \\
\end{tabular} & 7 & 7 & 7 & 7 & & & 7 & 7 & 7 & & 7 & 7 & 7 & 7 & 7 & & 7 & 7 & 7 & 7 & 7 & & & & 5 & & 55 & 5 & & & & & & & & & \\
\hline E & & & & & 10 & 10 & 10 & \begin{tabular}{|l|l|}
0 & 10 \\
\end{tabular} & & & & & & & & & & & 10. & 10 & 10 & 10 & 10 & & & & 10. & & 10 & 10 & 101 & & \begin{tabular}{l|l}
0 & 1 \\
\end{tabular} & & 0.16 & 10 & 10 & 10 & & & 10 & 10 & 101 & \begin{tabular}{l|l}
10 & 1 \\
\end{tabular} & 10 \\
\hline 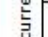 & & & & & & & & & & & & & & & & & & & & & & & & & & & & & & & 7 & & & & & & & & & & & & & & \\
\hline s & & & & & & & & & & & & & & & & & & & & & & & & & & & & & & 13 & & & & & & & & & & & & & & & \\
\hline
\end{tabular}

Fig. 20. Task allocation [11] to the Employees in ST-5

\begin{tabular}{|c|c|c|c|c|c|c|c|c|c|c|c|c|c|c|c|c|c|c|c|c|c|c|c|c|c|c|c|c|c|c|c|c|c|c|c|}
\hline \multicolumn{35}{|c|}{ Task allocation to employees in ST-5 type schedule Part- 2} & \\
\hline \multicolumn{4}{|c|}{ Month13 } & \multicolumn{4}{|c|}{ Month14 } & \multicolumn{4}{|c|}{ Month15 } & \multicolumn{4}{|c|}{ Month16 } & \multicolumn{4}{|c|}{ Month17 } & \multicolumn{4}{|c|}{ Month18 } & \multicolumn{4}{|c|}{ Month19 } & \multicolumn{4}{|c|}{ Month20 } & \multicolumn{4}{|c|}{ Month21 } \\
\hline 49 & 50 & 51 & 52 & 53 & 54 & 55 & 56 & 57 & 58 & 59 & 60 & 61 & 62 & 63 & 64 & 65 & 66 & 67 & 68 & 69 & 70 & 71 & 72 & 73 & 74 & 75 & 76 & 77 & 78 & 79 & 80 & 81 & 82 & 83 & \\
\hline 1 & 1 & 1 & 1 & 1 & 1 & 1 & 1 & 1 & 1 & 1 & 1 & 1 & 1 & 1 & 1 & 1 & 1 & 1 & 1 & 1 & 1 & 1 & 1 & 1 & & & 1 & 1 & 6 & & & & & & \\
\hline 6 & 6 & 6 & 6 & 6 & 6 & 6 & 6 & & & & & 6 & 6 & 6 & 6 & 6 & 6 & 6 & 6 & 6 & 6 & 6 & 6 & 6 & & & 6 & 6 & 6 & 6 & 6 & 6 & 6 & 6 & \\
\hline 8 & 8 & 8 & 8 & 8 & 8 & 8 & 8 & 8 & 8 & & & 8 & 8 & 8 & 8 & 8 & 8 & 8 & 8 & 8 & 8 & 8 & & & & & 8 & 8 & 8 & 8 & 8 & 8 & & & \\
\hline 9 & 9 & 9 & 9 & 9 & 9 & 9 & 9 & & & & & 9 & 9 & & $\mathrm{~T} 12$ & & 9 |- & $\mathrm{T} 16$ & 9 & & & & & & & & 9 & 9 & 9 & 9 & 9 & & & & \\
\hline & & & & & & & & & & & & & & & 3 & 3 & $3 \Gamma$ & 3 & 3 & 3 & & & & & & & & & T17 & & & & & & \\
\hline 3 & & & & & & & & & & & & & & & & & & & & & 3 & & & & & & 3 & 3 & 5 & 6 & 6 & 6 & 6 & 6 & \\
\hline 2 & 2 & & & & & & & & & & & 2 & 2 & 2 & 2 & 2 & 2 & 2 & 2 & 2 & & & & & & & 2 & 2 & 2 & 2 & 2 & 2 & 2 & 2 & \\
\hline 0 & T14 & 0 & 0 & & & & & & & & & & & & & & & & & & & & & & & & & & & & & & & & \\
\hline 4 & 4 & 4 & 4 & 4 & 4 & 4 & 4 & 4 & & & & 4 & 4 & 4 & 4 & 4 & 4 & 4 & 4 & 4 & & & & 4 & & & & & & & & & & & \\
\hline 5 & 5 & 5 & 5 & 5 & 5 & 5 & 5 & 5 & 5 & 5 & 5 & 5 & 5 & 5 & 5 & 5 & 5 & 5 & 5 & 5 & 5 & 5 & 5 & 5 & 5 & 5 & 5 & 5 & 5 & 5 & 5 & 5 & & & \\
\hline & & & T15 & & & & & & & 9 & 9 & & & & 1 & 1 & 1 & & & & & & & & & & 9 & & & & 9 & 0 & & & \\
\hline 10 & 10 & 10 & 10 & 10 & & 2 & & & & 3 & 3 & & & & & & & & & & & & & & & & & & & 7 & 7 & 7 & 7 & 7 & \\
\hline 7 & 7 & 7 & 7 & & & 10 & & & & & & 7 & 7 & 7 & 7 & 7 & 7 & & & & & & & & & & 7 & 7 & 7 & 4 & 4 & 4 & 4 & & \\
\hline & & 10 & 10 & 10 & 10 & $|10|$ & & & & & & & & & 10 & 10 & & & & & & & & & & & 10 & 10 & 10 & 10 & 10 & & & & \\
\hline
\end{tabular}

Fig. 21. Task allocation [11] to the Employees in ST-5

work. The paper [27 gives help to push in Analysis, mathematical modeling and algorithm for software project scheduling using BCGA as this study oriented article is supported and inspired by Michael Rinehart, Vida Kianzad and Shuvra S. Battacharya [38] for getting the knowledge of the concerned domain in the future direction of BCGA.

The paper [14] gives idea about the defect classification by DTL. The brief idea is put forward by Dinesh, Santosh and Shabina Sayyad [14] how one should make the classification in the software of defect as metrics in the software testing and test planning which is one the goal of the STQA (Software Testing and Quality Analysis). The work done by Dinesh, Digambar and Santosh [15] gives touch to the another part of project management in software i.e. risk management. The team showed how one should predict the risk in software project by Bayesian learning. The learning gives output to PM to manage the risks in the project according to the type of project. SPM consist one of the important phase i.e. SCE (Software Cost Estimation). PM has tough tasks to do about to put the minimum cost at the cost of the defects found in the project. If the developers do the proper identification and classifi- 
cation of defects after and before of the every phases, milestones or sub module, it will be easy for making remedies and solutions for overcoming form the wastage of money. This has been shown by the group of Jawahar, Santosh and Dinesh [16]. Another important part of our future work is training of employee and their education which is another upheld task of scheduling. The same thing in technical and management point of view is shown by Dinesh and Vipul in Organizational Behavior \& Its improvement by Software Engineering Process Models Training paper [19]. The paper written by Dinesh Zende and Dinesh [13] gives idea regarding powerful GA which can be used for optimisation. Reshma [44] tried to develop Scheduling project with Random and time-dependent activity. She got the good results and attempted to make the outcome applicable to find the critical path in random and time dependent project scheduling problem, But, the result achievement is average compare to the intellectual and rich article [29] of Wei Huang and Lixin Ding. Another article named Śoftware Project Planning Using Ant Colony Optimization (SPP-ACO)éxpressed by Kishor Vitekar and Dinesh [33] gives better result than the Alba's result [21] in terms of cost but the duration of project is approximately and equal to the duration result of Alba. Urmila [42| reviewed and classified the literature on personnel scheduling problem. The objective was to identify broad classication, compare different methods and identify the future research directions.

\section{REFERENCES}

[1] PMBOK A Guide to the Project Management Body of Knowledge. www.amazon.com.

[2] PMI India PMBOK Guide and Standards.

[3] Software asset management. www.software.one.com.

[4] Felician ALECU. Management of software development projects. Economics of Knowledge, 33, 2011.

[5] Konduru Sivaramakrishnan Anandasivam Gopal. On vendor preferences for contract types in offshore software projects: The case of fixed price vs. time and materials contracts.

[6] Rajib Mall Bob Hughes, Mike Cotterell. Software Project Management(SEI). Tata McGraw-Hill Education Pvt. Ltd, BookVistas (New Delhi, DEL, India), April 2011.

[7] Barry Bohem. Software Engineering economics. Prentice Hall PTR, New Jersey.

[8] Frederick P. Brooks Jr. The Mythical ManMonth. AddisonWesley, 1995.

[9] Tao Zhang Carl K. Chang, Mark. J. Christensen. Genetic algorithms for project management. annals of Software Engineering, 2001.

[10] C.K. Chang and M. Christensen. A net practice for software project management. 1999.

[11] Bob Hughes \& Mike Cotterell. Software Project Management. McGraw-Hill Publishing Company, BookVistas (New Delhi, DEL, India), 1999.

[12] Amit Kurmi Dinesh B. Hanchate. Design and analysis of algorithm. Technical Publication, Pune, 2007.

[13] Dinesh A. Zende Dinesh B. Hanchate. Clustering and classification of text by selection process and optimisation by genetica algorithm. ACITS PINNACLE'08, National Conference on Systematics, Informatics, Cybernatics, March 2008.

[14] Santosh A. Shinde Dinesh B. Hanchate, Shabina Sayyad. Defect classification as problem classification for quality control in the software project management by DTL. IEEE International Conference on Computer Engineering and Technology - ICCET, 2010.

[15] Digambar M. Padulkar Dinesh B.Hanchate, Santosh A. Shinde. Impact of risk factors in risk management by bayesian learning. ACM Proceedings of International Conference on Advances in Computing ICAC2008., feb. 2008. http://itfrindia.org/2011ICCIC/program.php.

[16] Yogesh A. Thorat Dinesh B.Hanchate, Rajaram H.Ambole. Optimization of university course timetabling problem (uctp) using genetic algorithm: A survey. IEEE ICCIC2011, 2011. http://itfrindia.org/2011ICCIC/program.php.

[17] Dr. Rajankumar S. Bichkar Dinesh.B.Hanchate. Software project scheduling by AGA. International journal of computer Applications, 96, June 2014.

[18] Dr. Rajankumar S. Bichkar Dinesh.B.Hanchate. SPS by combination of crossover types and changeable mutation SGA. International journal of computer Applications, 94, May 2014.

[19] Vipul V. Bag Dinesh.B.Hanchate. Organizational behavior and its improvement by software engineering process models training. proceedings of National Conference on Best practices in Engineering Education ( NCBPEE 2007), feb 2007.

[20] S. Sahani E. Horowitz. Fundamentals of Computer Algorithms. Galgotia Publications, 1999.

[21] J. Franciso Chicano Enrique Alba. Sofware project management with gas. SceinceDirect, Information Sciences, 177, 2007.

[22] Jeff G. http://www.excella.com/blog/fixed-price-vs-time-andmaterials-better-software-development-contract-type/. 2002.

[23] M.R Garey and D.S. Johnson. Computers and Intractability. A Guide to the Theory of NP-Completeness. W. H Freeman and Company, New york, 1979.

[24] David Barnes Mark McCarville George G. Mitchell, Diarmuid ODonoghue. Generepair a repair operator for genetic algorithms. Proceedings of the GECCO-2003 Late Breaking Papers, July 2003.

[25] D.E. Goldberg. Genetic Algorithm in Search, Optimiza-tion and Machine Learning. Addison-Wesley, 1989.

[26] Dinesh B. Hanchate Gyankamal J. Chhajed, Mangesh Kulkarni. Object Oriented Modelling Desing. Pragati, Pune, 2007.

[27] Dinesh Bhagwan Hanchate. Analysis, mathematical modeling and algorithm for software project scheduling using BCGA. IEEE Intelligent Computing and Intelligent Systems (ICIS), Oct. 2011

[28] J.H. Holland. Genetic Algorithms. Scientific american edition, 1992.

[29] Wei Huang and Lixin Ding. Project-scheduling problem with random time-dependent activity duration times. IEEE Transactions on engi-neering management, May 2011.

[30] Pankaj Jalote. Software project management in practice. Addison Wesley, New york, 2004.

[31] Pankaj Jalote. An Integrated Approach to Software Engineering. Narosa publishing House, New york, 2005.

[32] Stephen H. Kan. Metrics and Models in Software Qaulity Engineering. Low price edition edition, 2006.

[33] Dinesh B Hanchate Kishor N Vitekar. Software project planning using ant colony optimization (spp-aco). International Journal of Computer Science And Technology, Oct 2013. www.ijcst.com. 
[34] Ying-Hong Liao and Chuen-Tsai Sun. An educational genetic algorithms learning tool. IEEE transactions in Education, 2001.

[35] Walt Lipke. Earned schedule application to small projects. PM WORLD TODAY, 2011.

[36] Gangadharrao Soundalyarao "G. S." Maddala. LimitedDependent and Qualitative Variables in Econometrics. Cambridge University Press, Cambridge, UK, 1983.

[37] Duncan McGillivray. User manual for garefl. http://www.reflectometry.org/danse.

[38] Vida Kianzad Michael Rinehart and Shuvra S. Battacharya. Modular genetic algorithm for scheduling task garphs. Technical report UMIACS-TR-2003-66.

[39] Vassil Smarkov Milena Karova, Julka Petkova. A genetic algorithm for project planning problem. International Scientific Conference Computer Science, 2008.

[40] Tom M. Mitchell. Machine Learning. The McGrawHill Companies, 1997

[41] Himanshu Bhalchandra Dave Parag Himanshu Dave. Design and Analysis of Algorithms. Pearson Education, 2008.

[42] Urmila Shrikant Pawar and Dinesh Bhagwan Hanchate. Literature review on personnel scheduling. International Journal of Computer Engineering and Technology (IJCET), Spt 2013. www.iaeme.com.

[43] Roger S. Pressman. Software Engineering: A practitioners Ap-proach. McGrawHill, Inc., New york, 1992.

[44] Yogita D. Sinkar Reshma R. Nazirkar, Dinesh B. Hanchate. Scheduling project with random and time-dependent activity. IEEE International conference on computation of power, energy, information and communication (ICCPEIC), April 2014.

[45] Anita Rosen. Effective IT Project Management: Using Teams to Get Projects Completed on Time and Under Budget. PHI, BookVistas (New Delhi, DEL, India), 2008.

[46] Priya V. Patil S.D. Joshi Santosh A. Shinde, Chudaman D. Sukate. Project management by dtl. National Conference on Artificial Intelligence (NCAI 2007), June 2007. xa.yimg.com/kq/groups/18445735/80921961/name/A.

[47] Kathy Schwalbe. It pm book. www.pmtext.com.

[48] Santosh A. Shinde. Software team formation for software project management by neural networks \& DTL. International Conference on Innovative Computing, Information and Communication Technology (ICICT09), Dec. 2009.

[49] I Sommerville. Software Engineering. Addison Wesley, New york, 1998.

[50] Du Zhang and Je rey J.P. Tsai. Machine learning and software engineering. IEEE, Proceedings of the 14th International Conference on Tools with Arti cial Intelligence, 2002.

[51] Gabrielle Zimmerman. Contract types for software development. TurnLevel.

Dinesh Bhagwan Hanchate Birth Place :- Solapur, B.E. Computer from Walchand College of Engineering, Sangli (1995), Lecturer in Gangamai College Of Engineering,Dhule (1995-96), Lecturer in S.S.V.P.S.s B.S.D. College Of Engineering,Dhule In Computer \& IT deptt ( 1996-2005), M.Tech. Computer from Dr. Babasaheb Ambedkar Technological University, Lonere(2002-05), Currently Asst. Prof. Computer Engineering, former H.O.D. ( Computer \& IT ) in Vidya pratishthans College Of Engineering, Baramati , currently doing research (SGGSs Institute of Technology and
Engg, Nanded affiliated to SRTMU,Nanded) under the guidance of Dr. Bichkar R.S. ,G.H. Raisonis College Of Engineering and Management,Wagholi,,Pune.

obtained his BE and ME degrees in electronics from the SGGS institute of Engineering and Technology, Nanded, in 1986 and 1990 respectively, and his PhD from IIT Kharagpur in 2000. He served as faculty in the computer engineering and electronics engineering departments in the SGGS Institute of Engineering and Technology from 1986 to 2007, and is presently a professor in the Department of Electronics and Telecommunication Engineering, G H Raisoni College of Engineering and Management, Pune. He has taught several subjects at undergraduate and postgraduate level, including programming in $\mathrm{C}$ and $\mathrm{C}++$, data structures computer algorithms, microprocessors, information technology, DBMS and electronic devices and circuits. His research interests include application of genetic algorithms to various search and optimization problems in electronics and computer engineering. 